Preprint typeset in JHEP style - HYPER VERSION

MCTP-04-46, YITP-04-41

SPHT-T-04/102, NEIP-04-04

hep-th/0408171

\title{
Covariant One-Loop Amplitudes in $\mathrm{D}=11$
}

\author{
Lilia Anguelova \\ Michigan Center for Theoretical Physics, University of Michigan \\ Ann Arbor, MI 48109-1120, USA, \\ anguelov@umich.edu \\ Pietro Antonio Grassi \\ C.N. Yang Institute for Theoretical Physics, SUNY at Stony Brook \\ Stony Brook, NY 11794-3840, USA, \\ and \\ DISTA, Università del Piemonte Orientale, \\ Piazza Amborsoli, 115100 Alessandria, Italy \\ pgrassi@insti.physics.sunysb.edu
}

\author{
Pierre Vanhove \\ CEA/DSM/SPhT, URA au CNRS, CEA/Saclay, \\ F-91191 Gif-sur-Yvette, France \\ vanhove@spht.saclay.cea.fr
}

\begin{abstract}
We generalize to the eleven-dimensional superparticle Berkovits' prescription for loop computations in the pure spinor approach to covariant quantization of the superstring. Using these ten- and eleven-dimensional results, we compute covariantly the following one-loop amplitudes: $C \wedge X_{8}$ in M-theory; $B \wedge X_{8}$ in type II string theory and $\mathcal{F}^{4}$ in type I. We also verify the consistency of the formalism in eleven dimensions by recovering the correct classical action from tree-level amplitudes. As the superparticle is only a first approximation to the supermembrane, we comment on the possibility of extending this construction to the latter. Finally, we elaborate on the relationship between the present BRST language and the spinorial cohomology approach to corrections of the effective action.
\end{abstract}

KEYWORDS: pure spinors, superparticle, eleven dimensions. 


\section{Contents}

1. Introduction 2

2. From $\kappa$-symmetry to BRST symmetry for 11d superparticle 4

3. Supergravity in 11 dimensions and vertex operators 8

3.1 Vertex Operators and $N=1, D=11$ Supergravity 8

3.2 Coupling of the action to a background 10

4. Tree-level amplitudes 11

4.1 Path integral definition for the tree-level measure 11

4.2 Measure of integration for the pure spinor $\lambda \quad[12$

4.3 Tree-level action from scattering amplitudes 14

5. Definition of the one-loop amplitude 16

5.1 Measure for the conjugate momentum $w_{A}$

5.2 A different representation of the $b_{B}$ ghost 19

5.2.1 Action of the $b_{B}$ ghost on vertex operators 20

6. One loop amplitudes 22

$6.1 B \wedge X_{8}$ in ten dimensions 23

$6.2 C_{3} \wedge X_{8}$ in eleven dimensions 26

6.3 Open string amplitudes in ten dimensions 28

7. Equivalence between spinorial and BRST cohomology 30

7.1 Deformations and cohomology 30

7.2 Spinorial cohomology from an extended BRST operator 32

8. Recapitulation and discussion 35

丹. Fierz identities and Gamma matrix manipulations in 11d 36

B. $\mathrm{SO}(8)$ parametrization of the 11d Pure Spinors 38 


\section{Introduction}

Since the discovery of the web of dualities that relate the five 10-dimensional string theories with each other and with 11-dimensional supergravity [1], it has been realized that perturbative and nonperturbative effects in ten-dimensions are encoded in the effective action of an underlying 11-dimensional theory. In particular, both the fundamental string and the D2-brane originate from the 11-dimensional supermembrane. Although the relationship between this latter M2-brane and the eleven-dimensonal supergravity fields is what one would expect from a fundamental object and its long-range fields [2], it has turned out that there is no gap between the massless and massive exitations of the membrane [3, [1. Nevertheless one can consistently define vertex operators for the massless fields [5] and study the corresponding scattering amplitudes. ${ }^{1}$ In full generality, this is still a task beyond reach. However in the limiting case of the superparticle, i.e. suppressing the transverse fluctuations of the supermembrane, several successful computations have been performed in the light cone gauge. More precisely, the 11d four-graviton scattering was computed to one and two loops in [7, 8] and [9] respectively. Of course, these supergravity amplitudes are UV divergent but the lack of knowledge about the microscopic degrees of freedom can be compensated by information provided from various dualities. This is how, for example, the one-loop generated eleven-dimensional $R^{4}$ term was "renormalized" in [7]. The important lesson from the point-particle limit of the membrane is that quantum supergravity in 11d does indeed reproduce the correct form of certain terms in the effective action of M-theory, for example the $11 \mathrm{~d}$ one-loop $R^{4}$ term which gives rise to both the tree level and the one-loop $R^{4}$ terms in the effective superstring action.

However, the light cone gauge, used in the above works, has its drawbacks in that it is not suitable for computing all possible amplitudes in a generic background and also it is less efficient in organizing the calculations, in particular making manifest the cancellations between different contributions, than a covariant formulation would be. The first steps towards a full covariant description were made in [10], based on the recent progress in the covariant formulation of $10 \mathrm{~d}$ superstring theory [11]. The idea is to construct a supermembrane action, alternative to [2], which reduces to the ten-dimensional pure spinor description of the type IIA superstring after double dimensional reduction. This action is invariant under a BRST transformation generated by the same type of operator $Q=\lambda^{A} d_{A}$ as the one used in [11] for the case of superstrings. So again one can define vertex operators via the cohomology of $Q$. Using this covariant BRST formulation, the

\footnotetext{
${ }^{1}$ On the other hand, vertex operators, analogous to the massive superstring states, are not expected to exist due to the continuous spectrum of the supermembrane. Instead, the membrane excitations are interpreted as being related to multi-particle (rather than one-particle) states in the Matrix Theory proposal of [6].
} 
general form of the vertex operators relevant for the supermembrane theory massless excitations, namely the graviton $g_{M N}$, gravitino $\psi_{M}^{A}$ and three-form $C_{M N P}$, was obtained in [10], although some of the superfields in that expression remained undetermined. Clearly, these vertices can be reduced to those of the 11d superparticle by setting to zero the transverse fluctuations of the supermembrane.

Although [10] gives a tree-level prescription for integration over the zero modes of the pure spinor ghosts $\lambda^{A}$ and their conjugate momenta $w_{A}$ it does not derive in general the measure for this integration and so leaves performing loop computations out of reach. The same problem was resolved only very recently for the pure spinor formulation of 10dimensional string theory [12]. The crucial ingredient was the construction of a composite field playing a role analogous to the one of the NSR $b$-antighost. This is very nontrivial in Berkovits' formalism as the only field with negative ghost number, $w_{A}$, appears always in combination with $\lambda^{A}$, i.e. only in expressions with ghost number zero. This problem was overcome in [12 by introducing picture-changing operators and defining the $b$-ghost via $\{Q, b\}=T Z$, where $T$ is the energy-momentum tensor and $Z$ is a picture raising operator with ghost-number 1. As pointed out in that work, the necessity of picture-changing operators should have been anticipated, since the pure spinor ghosts are world-sheet bosons with zero modes similarly to the NSR $(\beta, \gamma)$-ghosts.

Unlike the superstring, the membrane world-volume theory is not conformally invariant. But, as in the string case, it is still reparametrization invariant. Therefore one should expect an analogue of the $b$-ghost in eleven dimensions as well. In this paper we show that the 10-dimensional covariant rules for superstring loop calculations of [12] can be extended to the case of the 11-dimensional superparticle. In fact, we construct the $11 \mathrm{~d}$ generalization of the "antighost" field $b_{B}$ in a form that is also valid for string theory. The advantage of our different, though equivalent to the one in [12], representation of $b_{B}$ is that it simplifies the prescription for one loop computations given there. Our construction of covariant one loop amplitudes, as well as the light-cone calculations of [8], is based on the string-inspired first quantized approach to field theory developed in [13, 14, [7, 9]. Namely, one inserts vertex operators, describing the external fields, on the world-line of the particle circulating in the loop and integrates over that world-line. For that purpose we derive the 11d superparticle vertex operator and show that it is consistent with the general form obtained in $[10] .^{2}$

Using the superstring formalism of [12] and the 11d one of this paper, we compute covariantly several one-loop amplitudes: the terms $B \wedge X_{8}$ in IIA/B string theory and $C_{3} \wedge X_{8}$ in $11 \mathrm{~d}$ supergravity and also the supersymmetric $\mathcal{F}^{4}$ in type I (meaning all

\footnotetext{
${ }^{2}$ Recall that, as we already mentioned, many of the superfields in that general form are actually still unknown.
} 
three cases: four gluons; two gluons and two gluinos; four gluinos), always finding the correct answers. We also show that the tree-level scattering amplitudes in $11 \mathrm{~d}$ reproduce completely the two-derivative action of eleven-dimensional supergravity [15]. We view our results as a strong confirmation of the viability of the pure spinor approach to covariant quantization.

Finally, we elaborate on the relashionship/equivalence between pure spinor cohomology and the spinorial cohomology developed in [16, 17, 18, 19], recovering the latter from an extended BRST operator similarly to the construction of 21 that relaxes the pure spinor constraint.

We conclude with some remarks about a possible extension of the present computation to the M2-brane.

\section{From $\kappa$-symmetry to BRST symmetry for 11d superparticle}

To introduce basic notions, we review in the current section the path leading from the $\kappa$ symmetric superparticle action of [25] to the action of Berkovits [26], essentially following [28]. We also explain how the tree-level measure in the pure spinor formalism, introduced initially ad hoc, maps to the light-cone measure.

The Brink-Schwarz super-Poincaré invariant point-particle action is

$$
\begin{gathered}
S_{\text {covariant }}=\int d \tau\left(P_{M} \Pi^{M}-\frac{1}{2} e P_{M} P^{M}\right), \\
\Pi^{M}=\dot{X}^{M}-i \bar{\Theta} \Gamma^{M} \dot{\Theta}
\end{gathered}
$$

where $M=0, \ldots, 10, \tau$ parametrizes the superparticle worldline, $\Theta^{A}$ are Majorana spinors with $A=1, \ldots, 32$, e denotes the worldline einbein and the indices of $P^{M}$ are lowered by the flat metric. This action is invariant under global supersymmetry,

$$
\delta_{\epsilon} \Theta=\epsilon ; \quad \delta_{\epsilon} X^{M}=i \bar{\epsilon} \Gamma^{M} \Theta ; \quad \delta_{\epsilon} P_{M}=\delta_{\epsilon} e=0
$$

and local $\kappa$-symmetry,

$$
\delta_{\kappa} \Theta=i P_{M} \Gamma^{M} \kappa ; \quad \delta_{\kappa} X^{M}=i \bar{\Theta} \Gamma^{M} \delta_{\kappa} \Theta ; \quad \delta_{\kappa} e=4 e \dot{\bar{\Theta}} \kappa ; \quad \delta_{\kappa} P_{M}=0 .
$$

It is also invariant under local reparametrizations.

Using $\kappa$-symmetry one can eliminate half of the components of $\Theta$. This is easily seen by choosing the light-cone gauge [8]

$$
X^{+}=x^{+}+p^{+} \tau ; \quad \Gamma^{+} \Theta=0
$$


where

$$
X^{ \pm}=\frac{1}{\sqrt{2}}\left(X^{0} \pm X^{10}\right) ; \quad \Gamma^{ \pm}=\frac{1}{\sqrt{2}}\left(\Gamma^{0} \pm \Gamma^{10}\right), \quad\left\{\Gamma^{+}, \Gamma^{-}\right\}=1 .
$$

The light-cone gauge action used by Brink and Schwarz [25] is

$$
S_{l . c .}=\int d \tau\left(\dot{X}^{M} P_{M}+\frac{1}{2} \dot{S}^{\alpha} S_{\alpha}-\frac{1}{2} e P^{M} P_{M}\right),
$$

where $S^{\alpha}=\sqrt{p^{+}}\left(\Gamma^{-} \Theta\right)^{\alpha}$ are the sixteen surviving $\mathrm{SO}(9)$ components of $\Theta$. In this gauge half of supersymmetry is realized linearly and the other half - nonlinearly:

$$
\begin{aligned}
\delta_{\varepsilon} S^{\alpha} & =\sqrt{p^{+}} \varepsilon^{\alpha} ; \quad \delta_{\epsilon} X^{M}=0 ; \quad \delta_{\varepsilon} P_{M}=\delta_{\varepsilon} e=0 \\
\delta_{\eta} S & =\frac{i}{\sqrt{p^{+}}} P_{M} \Gamma^{M} \Gamma^{+} \eta ; \quad \delta_{\eta} X^{M}=-\frac{2}{\sqrt{p^{+}}} \bar{\eta} \Gamma^{M} \Gamma^{+} S ; \quad \delta_{\varepsilon} P_{M}=\delta_{\varepsilon} e=0,
\end{aligned}
$$

where the parameter of the former, $\varepsilon$, satisfies $\Gamma^{+} \varepsilon=0$ and the parameter of the latter $-\Gamma^{+} \eta \neq 0$. Note also that in order to preserve $\Gamma^{+} \Theta=0$ one has to combine a linear supersymmetry transformation with a $\kappa$-symmetry one.

The wavefunction of the system carries $\mathrm{SO}(9)$ vector and fermionic degrees of freedom and can be expanded on the basis of physical states: the metric $|I J\rangle, 3$-form $|I J K\rangle$ and gravitino $|\alpha I\rangle$ (see [8] for details), where $I, J, K=1, \ldots, 9$. So the superspace is reduced from 32 original Grassmann coordinates to 16 free ones, $S_{\alpha}$, satisfying the Clifford relations $\left\{S_{\alpha}, S_{\beta}\right\}=\delta_{\alpha \beta}$. At tree level in string expansion, they can be organized into $S O(8)$ spinor representations $S_{\alpha}=\left(\xi_{a}, \xi_{a}^{\dagger}\right)$ and the tree level measure can be defined by the path integral formula

$$
\left\langle\xi_{a_{1}} \ldots \xi_{a_{8}}\right\rangle_{g=0}=\int d^{8} \xi \prod_{i=1}^{8} \xi_{a_{1}} \ldots \xi_{a_{8}}=\epsilon_{a_{1} \ldots a_{8}} .
$$

Accordingly, the states $|I J\rangle,|I J K\rangle$ and $|\alpha I\rangle$ are given in terms of $S O(8)$ representations obtained by acting with $\xi_{a}$ on the vacuum defined by $\xi_{a}^{\dagger}|0\rangle=0$ with $a \neq 0$. This agrees with the fact that at tree level only 8 zero modes coming from $\xi_{a}$ have to be saturated ( $\xi_{a}^{\dagger}$ play the role of conjugate momenta, i.e. $\left\{\xi_{a}, \xi_{b}^{\dagger}\right\}=\delta_{a b}$ ). Alternatively, we can write the one loop measure in a completely $S O(9)$ covariant way by saturating both the zero modes of $\xi_{a}$ and those of $\xi_{a}^{\dagger}$ :

$$
\left\langle S^{\alpha_{1}} \cdots S^{\alpha_{16}}\right\rangle_{g=1}=\int d^{16} S \prod_{i=1}^{8} S^{a_{1}} \cdots S^{a_{16}}=\epsilon^{\alpha_{1} \cdots \alpha_{16}}
$$

In order to compare (2.4) with the pure spinor approach, one adds two new doublets $\left(p_{A}, \theta^{A}\right)$ and $\left(w_{A}, \lambda^{A}\right)$, of anticommuting and commuting variables respectively, which are 
not related to the light-cone fermions $S^{\alpha}$. Then one defines

$$
\hat{d}_{A}=p_{A}+\frac{1}{2} P_{M}\left[\Gamma^{M}\left(\theta+\frac{1}{\sqrt{p^{+}}} \Gamma^{+} S\right)\right]_{A},
$$

which satisfies

$$
\left\{\hat{d}_{A}, \hat{d}_{B}\right\}=-\frac{i}{2 p^{+}} P_{M} P^{M}\left(\Gamma^{+}\right)_{A B} .
$$

In deriving this relation we have used that $S^{\alpha}$ are their own canonical conjugates (i.e., they satisfy $\left.\left\{S_{\alpha}, S_{\beta}\right\}=\delta_{\alpha \beta}\right)$. The first-class constraints $d_{A} \approx 0^{3}$ are associated with the BRST operator

$$
Q^{\prime}=\lambda^{\prime A} \hat{d}_{A} ; \quad \lambda^{\prime} \Gamma^{+} \lambda^{\prime}=0
$$

and the gauge-fixed action

$$
S_{c o v}=\int d \tau\left(P_{M} \dot{X}^{M}-\frac{1}{2} e P_{M} P^{M}+\dot{\theta}^{A} p_{A}+\frac{1}{2} \dot{S}^{\alpha} S_{\alpha}+\dot{\lambda}^{\prime A} w_{A}^{\prime}\right) .
$$

In order to bring the BRST charge (2.10) into the form of Berkovits' BRST charge

$$
Q=\lambda^{A} d_{A} \quad \text { with } \quad \lambda \Gamma^{M} \lambda=0,
$$

we split the $\mathrm{SO}(10,1)$ commutting spinor $\lambda^{\prime}$ into its $\mathrm{SO}(9)$ components with respect to $\Gamma^{+}$as (see Appendix B for more details)

$$
\lambda^{\prime A}=\left(\lambda_{1}^{\prime \alpha}, \lambda_{2}^{\prime \alpha}\right) .
$$

With this decomposition the BRST charge (2.10) becomes

$$
Q^{\prime}=\lambda_{2}^{\prime \alpha} d_{2 \alpha}+\lambda_{1}^{\prime \alpha} \hat{d}_{1 \alpha}
$$

where we have used the subspace of $\mathrm{SO}(9)$ spanned by $\Gamma^{+} \lambda^{\prime}=0, \hat{d}_{2 \alpha}=d_{2 \alpha}$. Since $\lambda_{1}^{\prime \alpha}=\left(\Gamma^{+} \lambda^{\prime}\right)^{\alpha}=\left(\left(\Gamma^{+} \lambda^{\prime}\right)^{a},\left(\Gamma^{+} \lambda^{\prime}\right)^{\dot{a}}\right)$ is a null $\mathrm{SO}(9)$ spinor preserved by a $\mathrm{SO}(8)$ subgroup chosen to act on the indices $\alpha$, there is a similarity transformation 28 that sends the components $\left(\Gamma^{+} S\right)_{a}$ onto $\left(\Gamma^{+} d\right)_{a}$ :

$$
Q^{\prime} \rightarrow e^{-i S \Gamma^{+} d / \sqrt{p^{+}}} Q^{\prime} e^{i S \Gamma^{+} d / \sqrt{p^{+}}}
$$

and the components $\left(\Gamma^{+} S\right)_{\dot{a}}$ are re-absorbed by shifting $\left(\theta+\sqrt{p^{+}}-1 \Gamma^{+} S\right)_{\dot{a}} \rightarrow \theta_{\dot{a}}$ in (2.9). The similarity transformation has to preserve the null spinor nature of $\Gamma^{+} \lambda^{\prime}$ and one component of $\left(\Gamma^{+} S\right)^{a}$ is not gauged into $d_{a}$ by (2.13) but is shifted into an extra component

\footnotetext{
${ }^{3}$ Notice that $P_{M} P^{M} \approx 0$ is also a first class constraint.
} 
of $\theta$. As shown in Appendix $\mathrm{B}$ the components of $\lambda$ are identified with the components of $\lambda^{\prime}$ via $\lambda^{A}=\left(\lambda^{\prime \dot{a}}, \sqrt{v^{+}-s_{i} s^{i}}, s_{i}, 0\right)$ with $i=1, \cdots, 7$, which gives $16+8-1=23$ components.

The final result is the BRST invariant action

$$
S_{c o v}=\int d \tau\left(P_{M} \dot{X}^{M}-\frac{1}{2} P_{M} P^{M}+\dot{\theta}^{A} p_{A}+\dot{\lambda}^{A} w_{A}\right)
$$

where $\lambda^{A}$ satisfies the pure spinor constraint $\lambda^{A}\left(\Gamma^{M}\right)_{A B} \lambda^{B}=0 .{ }^{4}$ The BRST operator becomes $Q=\lambda^{A} d_{A}$, which is nilpotent only up to pure spinor constraints and gauge transformations $\delta w_{A}=\Lambda_{M}\left(\Gamma^{M} \lambda\right)_{A}$, where $\Lambda_{M}$ is a local gauge parameter. It is easy to check that $S_{c o v}$ is invariant under $\delta w_{A}$ and that also $\dot{Q}=0$. Notice that on-shell, the only non constant field is $X^{M}$. The action $S_{\text {cov }}$ is manifestly super-Poincaré invariant. In addition, the ghost field part of the Lorentz generators, $N^{M N}=\frac{1}{2} w \Gamma^{M N} \lambda$, as well as the ghost current $J=w_{A} \lambda^{A}$ are invariant under the gauge transformations of $w_{A}$.

The light-cone variables satisfy the zero mode saturation identity (2.7), which expresses the fact that the superparticle preserves half of the original supersymmetry. We will see now that it gets mapped into the tree level measure

$$
\left\langle\left(\lambda \Gamma^{M_{1}} \theta\right) \cdots\left(\lambda \Gamma^{M_{7}} \theta\right)\left(\theta \Gamma_{M_{1} \cdots M_{7}} \theta\right)\right\rangle_{g=0}=1
$$

in the pure spinor formalism. For that purpose, we work in the space with both species of variables and we define the vertex operator $U^{(3)}=\lambda^{A} \lambda^{B} \lambda^{C} A_{A B C}(\theta)$ by its expansion in terms of the background fields $C_{M N P}, g_{M N}$ and $\psi_{A M}$. Now, we use the expressions for $C_{M N P}, g_{M N}$ and $\psi_{A M}$ in terms of the light-cone $\xi_{a}$-expansion and so obtain a vertex operator of the form $U^{(3)}=\lambda^{A} \lambda^{B} \lambda^{C} A_{A B C}(\theta, \xi)$. In the same way we have to define a vertex operator $U^{(4)}$ (see [10] and next sections for details). Then one can show that

$$
\epsilon^{a_{1} \ldots a_{8}} \xi_{a_{1}} \ldots \xi_{a_{8}}=\left\langle U^{(3)}(\lambda, \theta, \xi) U^{(4)}(\lambda, \theta, \xi)\right\rangle_{g=0}
$$

where the correlator is taken only in the $\lambda, \theta$ space. This maps the measure for zero modes in the pure spinor approach into the measure factor for light-cone variables at tree level.

Concluding this section, we note that from the above light-cone discussion it follows that any correlator with more than sixteen fermions vanishes. This means that any treelevel correlators $\left\langle\lambda^{7+p} \theta^{9+q}\right\rangle$, with $p$ and $q$ positive integers, are zero. In other words,

${ }^{4}$ Note that this definition differs form Cartan's pure spinors, also used by P. Howe, since the latter obey in addition $\lambda^{A}\left(\Gamma^{M N}\right)_{A B} \lambda^{B}=0$. Interestingly enough, similar constraints, more precisely $\lambda^{A}\left(\Gamma^{M N}\right)_{A B} \lambda^{B} \Pi_{M}=0$, emerge in the quantization of the supermembrane 10 and the covariant description of D-branes [27]. Another obvious remark about the $11 \mathrm{~d}$ constraint $\lambda^{A}\left(\Gamma^{M}\right){ }_{A B} \lambda^{B}=0$ is that when written in terms of two 10d pure spinors it coincides exactly with the two conditions for the D0brane [27], which should be the case since the latter is related to the $11 \mathrm{~d}$ superparticle by dimensional reduction. 
the zero momentum cohomology of $Q$ (see (2.12)) is empty for $\lambda$-ghost number bigger than 7 , as shown by a direct computation in [10]. The same reasonning can be applied for the ten dimensional open string to infer that the zero momentum cohomology of the corresponding $Q$ is empty for $\lambda$-ghost number greater than 3 .

\section{Supergravity in 11 dimensions and vertex operators}

In this section we analyze the structure of the vertex operators for the particle limit of the eleven-dimensional supermembrane. First we derive them from BRST cohomology and explain their component field expansion. We also point out that they are consistent with the general expression with undetermined coefficients of the integrated covariant vertex of [10. Then by coupling to a nontrivial background we derive another version of the integrated and unintegrated operators, which will be useful in the rest of the paper.

From now on we use the following conventions in $11 \mathrm{~d}: M, N, \ldots-$ curved vector indices; $A, B, \ldots$ - curved spinor indices; $r, s, \ldots$ - flat vector indices; $a, b, \ldots$ - flat spinor indices. Also $Z^{\Omega} \equiv\left(X^{M}, \Theta^{A}\right)$. In $10 \mathrm{~d}$ we denote by $m, n, \ldots$ curved vector indices and by $\alpha, \beta, \ldots$ curved spinor indices. Finally $I, J, \ldots=1,2$ run over the spatial directions of the membrane and $\partial_{0}$ is a derivative w.r.t. the $\mathrm{M} 2$ worldvolume time.

\subsection{Vertex Operators and $N=1, D=11$ Supergravity}

The vertex operator for the 11d superparticle $V^{(0)}=\int d \tau \mathcal{V}^{(0)}$ at ghost number zero can be viewed as a deformation of the quantum action expanded around flat background. It emerges as the first non-trivial contribution

$$
S_{d e f}=S_{c o v}+\int d \tau \mathcal{V}^{(0)}+\mathcal{O}(V),
$$

where $\mathcal{O}(V)$ denotes higher terms in the expansion. In order that the action $S_{\text {def }}$ is still invariant under BRST symmetry, one has $\left\{Q, \mathcal{V}^{(0)}\right\}=\partial_{\tau} U^{(1)}$, where $U^{(1)}$ is a ghost number one vertex operator. The latter satisfies $\left\{Q, U^{(1)}\right\}=0$ by consistency.

Acting with the BRST operator $Q$ on a generic polynomial of the fields $P_{M}, X^{M}, d_{A}$, $\theta^{A}, \lambda^{A}$ and $N_{N R}=\frac{1}{2}\left(w \Gamma_{N R} \lambda\right)$, one finds that

$$
\mathcal{V}^{(0)}=P^{M}\left(g_{M N} P^{N}+E_{M}{ }^{A} d_{A}+H_{M}^{[N R]} N^{N R}\right)
$$

and

$$
U^{(1)}=\lambda^{A} E_{A}{ }^{r} E_{M}{ }^{s} P^{M} \delta_{r s},
$$

where $g_{M N}=E_{M}{ }^{r} E_{N}{ }^{s} \delta_{r s}, E_{M}{ }^{A}$ and $H_{M}^{[N R]}$ are superfields which satisfy the supergravity field equations. We also note that (3.2) is easily seen to be consistent with the light-cone vertices of [8]. 
The vertex operator $\mathcal{V}^{(0)}$ is defined up to a gauge transformation generated by total derivative terms, i.e. $\delta \mathcal{V}^{(0)}=\partial_{\tau} \Omega^{(0)}$. Correspondently, the vertex $U^{(1)}$ is defined up to BRST exact terms of the form $\delta U^{(1)}=\{Q, \Omega\}$. For the case at hand, the gauge transformations are given by

$$
\Omega=\Omega^{M} P_{M}+\Omega^{A} d_{A}+\Omega^{[M N]} N_{M N} .
$$

At leading order in the $\theta$ expansion their parameters are the following:

$$
\Omega^{M}=\xi^{M}+\mathcal{O}(\theta), \quad \Omega^{A}=\epsilon^{A}+\mathcal{O}(\theta), \quad \Omega^{[M N]}=\omega^{[M N]}+\mathcal{O}(\theta),
$$

where $\xi^{M}$ is the one of infinitesimal diffeomorphisms, $\epsilon^{A}$ - of infinitesimal supersymmetry transformations and $\omega^{[M N]}$ - of Lorentz gauge transformations (i.e., $S O(10,1)$ rotations).

The superfields $E=\left(E_{M}^{r}, E_{M}^{a}, E_{A}{ }^{r}\right)$ and $H_{M}^{[N R]}$, contained in the vertex operator $\mathcal{V}^{(0)}$, are comprised from the fields of the usual $11 \mathrm{~d}$ supergravity multiplet namely the graviton $e_{M}^{r}$, gravitino $\psi_{M}^{A}$ and 3 -form $C_{M N R}$. However, the 11d superparticle does not couple directly to the potential $C_{M N P}$, but only feels its field-strength $G=d C$. As a result, the tensor gauge transformations cannot be seen among the transformation rules given in (3.4).

The expansion of the superfields $E, H_{M[N R]}$ up to order $\mathcal{O}\left(\theta^{3}\right)$ in terms of the on-shell supergravity fields is the following [29, 30]:

$$
\begin{aligned}
E_{M}{ }^{r} & =e_{M}{ }^{r}+2 \bar{\theta} \Gamma^{r} \psi_{M}+\left(\bar{\theta} \mathcal{U}^{r \mid N P Q L}{ }_{M} \theta\right) G_{N P Q L}+\frac{1}{4} \bar{\theta} \Gamma^{r s t} \theta \omega_{M[s t]}+\mathcal{O}\left(\theta^{3}\right) \\
E_{M}{ }^{a} & =\psi_{M}{ }^{a}+\frac{1}{4} \omega_{M[r s]}\left(\Gamma^{r s} \theta\right)^{a}+\left(\mathcal{T}_{M}{ }^{N R S T} \theta\right)^{a} G_{N R S T}+\mathcal{O}\left(\theta^{3}\right) \\
E_{A}{ }^{r} & =-\left(\bar{\theta} \Gamma^{r}\right)_{A}+\mathcal{O}\left(\theta^{3}\right) \\
H_{M}^{[r s]} & =\omega_{M}^{[r s]}+\mathcal{O}\left(\theta^{2}\right)
\end{aligned}
$$

with

$$
\begin{aligned}
\mathcal{T}_{M}{ }^{N R S T} & =\frac{1}{288}\left(\Gamma_{M}{ }^{N R S T}-8 \eta_{M}^{[N} \Gamma^{R S T]}\right) ; \\
\mathcal{U}^{R \mid M N P Q L} & =\frac{1}{288}\left(5 \eta^{R[M} \Gamma^{N P Q L]}+8 \Gamma^{R[M N P} \eta^{Q] L}\right) .
\end{aligned}
$$

We note in passing that there are several different ways of obtaining the above expansions. The most well-known one is the gauge completion method used in [29]. A much easier alternative is the following: one can use the field equations and Bianchi identities to derive recursion relations for the component fields in the $\theta$ expansion of the supergravity 
superfields similarly to the case of 10-dimensional SYM 31. This procedure was applied recently to ten- and eleven- dimensional supergravity in 32 and 30 respectively. ${ }^{5}$

The superspace description of 11d supergravity, obtained by starting from the ghost number zero vertex operator $\mathcal{V}^{(0)}$, coincides with the superspace description given in [22, 23]. However, as shown in [24, 16], one can also use a formulation based on the constraint of the spinorial component of the four form $G_{4}=d C_{3}$. This constraint can be translated into a BRST closedness condition by considering another type of vertex operator $U^{(3)}=$ $\lambda^{A} \lambda^{B} \lambda^{C} A_{A B C}$. The superfield $A_{A B C}$ is defined up to gauge transformations $\delta A_{A B C}=$ $\Gamma_{(A B}^{M} \Xi_{C) M}$. By requiring that $U^{(3)}$ is BRST closed, one can easily derive the condition [10]

$$
D_{(A} A_{B C D)}=\Gamma_{(A B}^{M} A_{C D) M}
$$

where $A_{A B M}$ is an auxiliary superfield. ${ }^{6}$ This form of the unintegrated vertex operator is related to the integrated one for the supermembrane [10] $V_{\text {membrane }}^{(0)}=\int d^{3} x \mathcal{V}_{\text {membrane }}^{(0)}$, where

$$
\begin{gathered}
\mathcal{V}_{\text {membrane }}^{(0)}=\partial_{0} Z^{\Sigma}\left(A_{\Sigma \Gamma} \partial_{0} Z^{\Gamma}+C_{\Sigma}{ }^{A} d_{A}+\Omega_{\Sigma B}{ }^{A} w_{A} \lambda^{B}\right)+ \\
+\left(A_{\Sigma \Gamma \Lambda} \partial_{0} Z^{\Sigma}+C_{\Gamma \Lambda}{ }^{A} d_{A}+\Omega_{\Sigma \Lambda B}{ }^{A} w_{A} \lambda^{B}\right) \partial_{I} Z^{\Gamma} \partial_{J} Z^{\Lambda} \epsilon^{I J}+ \\
+A_{\Sigma \Gamma \Lambda \Xi} \partial_{I} Z^{\Sigma} \partial_{J} Z^{\Gamma} \partial_{K} Z^{\Lambda} \partial_{L} Z^{\Xi} \epsilon^{I J} \epsilon^{K L}+Y_{\Sigma B}{ }^{A} \partial_{I} Z^{\Sigma} w_{A} \partial_{J} \lambda^{B} \epsilon^{I J}
\end{gathered}
$$

By setting to zero the transverse fluctuations of the membrane $\partial_{I} Z^{\Sigma}=0$, one reduces (3.10) to the first line, which is indeed of the same form as the vertex given in (3.2).

\subsection{Coupling of the action to a background}

Recall that in curved space we denote by capital greek letters $\Omega, \Sigma, \ldots$ superspace curved indices in $11 \mathrm{~d}$ and by latin capital letters the tangent space indices. So $P_{M}$ and $d_{A}$ are the supersymmetric conjugate momenta to $Z^{\Omega}$. The superparticle action can be written as

$$
\begin{aligned}
S_{\text {curved }}= & \int d \tau\left(P_{M} E_{\Omega}{ }^{M} \partial Z^{\Omega}+d_{A} E_{\Omega}{ }^{A} \partial Z^{\Omega}-\frac{1}{2} \eta^{M N} P_{M} P_{N}+\right. \\
& \left.+w_{A}\left(\partial \lambda^{A}+\partial Z^{\Sigma} \Omega_{\Sigma,[M N]}\left(\Gamma^{M N}\right)_{A}^{B} \lambda_{B}\right)\right) .
\end{aligned}
$$

As always the connection couples to the ghost with a spin coupling. Clearly the equations of motion for the fields $x^{M}, \theta^{A}, p_{A}$ and $P_{M}$ are changed.

\footnotetext{
${ }^{5}$ We should mention yet another way of calculating the component fields in the $\theta$ expansion of superfields, namely the normal coordinates approach advocated for example in [33].

${ }^{6}$ These equations are readily solved by imposing the Wess-Zumino gauge $\theta^{A} A_{A B C}(x, \theta)=0$.
} 
In addition, the action (3.11) is no longer BRST invariant. This reflects itself in the lack of nilpotency of the BRST operator

$$
Q=\lambda^{A}\left(d_{A}+E_{A \Sigma} \partial Z^{\Sigma}\right)
$$

in curved space. The non-invariance of the action implies that $\dot{Q} \neq 0$. Imposing

$$
\dot{Q}=0, \quad\{Q, Q\}=0
$$

and using the pure spinor conditions, one ends up with the usual supergravity constraints on the supertorsion $T_{N R}{ }^{M}$ and field strenght $G_{M N R S}$ :

$$
\begin{array}{cl}
T_{B C}{ }^{A}=T_{A M}{ }^{N}=0, & T_{A B}^{M}=\left(\Gamma^{M}\right)_{A B}, \\
G_{A B C D}=G_{A B C M}=0, & G_{A B M N}=\left(\Gamma_{M N}\right)_{A B} .
\end{array}
$$

For later use we write down another version of the vertex operators (3.2) and (3.3),

$$
\begin{aligned}
V & =\int d \tau\left(\Pi_{M}+E_{M}^{A} d_{A}+H_{M}^{[P Q]} N_{P Q}\right) g^{M N}\left(\Pi_{N}+E_{N}^{B} d_{B}+H_{N}^{[P Q]} N_{P Q}\right) e^{i k \cdot X} \\
U^{(1)} & =\left(\Pi_{M}+E_{M}^{A} d_{A}+H_{M}^{[N R]} N_{N R}\right) E_{B}^{M} \lambda^{B} e^{i k \cdot X}
\end{aligned}
$$

obtained after integrating out $P^{M}$ in (3.11). Notice that the integrated vertex operator, expressed in this form, resembles the integrated vertex of the closed superstring.

\section{Tree-level amplitudes}

\subsection{Path integral definition for the tree-level measure}

It is convenient to rewrite (2.15) in a functional integral form for later comparison with one-loop amplitudes. In order to be able to integrate over the 23 independent pure spinor components, we have to define a suitable measure $\mathcal{D} \lambda$ which respects the pure spinor constraint and by gauge fixing the zero modes of $\theta^{A}$ and $\lambda^{A}$ (at three level and for flat worldvolume with marked points, there are no zero mode for $w_{A}$ and $d_{A}$ ). So we introduce the Lagrange multiplier $\chi_{I}$ and its BRST partner $\eta_{I}$, where $I=1, \ldots, 23$, as well as their respective conjugate momenta $\bar{\chi}^{I}$ and $\bar{\eta}^{I}$. Now the BRST operator $Q$ changes to $Q_{\text {new }}=Q+\chi_{I} \bar{\eta}^{I}$. We also introduce the constant gauge fixing parameters $C_{A}^{I}$ with the help of which the tree level functional integral becomes

$$
\begin{aligned}
\left\langle\prod_{i} U_{i}^{\left(n_{i}\right)}(\lambda, X, \theta)\right\rangle_{g=0}= & \int d^{11} X d^{11} P d^{32} \theta d^{23} \chi d^{23} \eta\left[d \lambda^{A_{1}} \ldots d \lambda^{A_{23}}\right] \\
& \left(\epsilon_{32} \mathcal{T}^{-1}\right)_{\left[A_{1} \ldots A_{23}\right]}^{\left(B_{1} \ldots B_{7}\right)} \prod_{j=1}^{7} \frac{\partial}{\partial \lambda^{B_{j}}}\left(e^{\left[Q_{n e w}, \eta_{I} C_{A}^{I} \lambda^{A}\right]}\right) \prod_{i} U_{i}^{\left(n_{i}\right)}(\lambda, X, \theta),
\end{aligned}
$$


where $\sum_{i} n_{i}=7$ and $\left(\epsilon_{32} \mathcal{T}^{-1}\right)_{\left[A_{1} \ldots A_{23}\right]}^{\left(B_{1} \ldots B_{7}\right)}$ is a unique tensor constructed in terms of $S O(10,1)$ Lorentz invariants. Integrating over $\chi_{I}$ and $\eta^{I}$, one can replace the exponential with the picture lowering operator $\prod_{I} C_{A}^{I} \theta^{A} \delta\left(C_{A}^{I} \lambda^{A}\right)$. This expression is manifestly independent of $C_{A}^{I}$ since the vertex operators $U^{\left(n_{i}\right)}$, as well as the measure, are BRST invariant. In the following section we will see that the measure $\mathcal{D} \lambda$ is indeed consistent with the pure spinor constraint. Computing the derivatives in the above expression, we end up with

$$
\begin{aligned}
\left\langle\prod_{i} U_{i}^{\left(n_{i}\right)}(\lambda, X, \theta)\right\rangle_{g=0}= & \int d^{11} X d^{11} P d^{32} \theta d^{23} \chi d^{23} \eta\left[d \lambda^{A_{1}} \wedge \cdots \wedge d \lambda^{A_{23}}\right] \\
& \left(\epsilon_{32} \mathcal{T}^{-1}\right)_{\left[A_{1} \ldots A_{23}\right]}^{\left(B_{1} \ldots B_{7}\right)} \prod_{j=1}^{7}\left(C_{B_{j}}^{I} \chi_{I}\right)\left(e^{\left[Q_{n e w}, \eta_{I} C_{A}^{I} \lambda^{A}\right]}\right) \prod_{i} U_{i}^{\left(n_{i}\right)}(\lambda, X, \theta) .
\end{aligned}
$$

Since the amplitude is independent of the gauge fixing parameters $C_{A}^{I}$, an easy way to compute it is to average over those parameters with a Gaussian-type measure such that $\int \mathcal{D} C=1$.

\subsection{Measure of integration for the pure spinor $\lambda$}

In this section we construct the bosonic ghost measure $[\mathcal{D} \lambda]$. The measure $[\mathcal{D} w]$ for the canonically conjugate momentum $w_{B}$ (recall that $\left[\lambda^{A}, w_{B}\right]=\delta^{A}{ }_{B}$ ) will be constructed in section 5.1 when discussing one-loop amplitudes.

The pure spinor condition

$$
\lambda \Gamma^{M} \lambda=0
$$

is a set of first class constraints and for constructing the measure of integration we use the same method as in [12]. Namely, from the zero mode prescription at tree-level (2.15) we construct a tensor $\mathcal{T}_{\left(\left(B_{1} \cdots B_{7}\right)\right)}^{\left[A_{1} \cdots A_{9}\right]}$, antisymmetric in the $A_{i=1, \ldots, 9}$ indices and traceless symetric in $B_{i=1, \ldots, 7}$, defined by

$$
\begin{aligned}
1 & =\left\langle\left(\lambda \Gamma^{m_{1}} \theta\right) \cdots\left(\lambda \Gamma^{m_{7}} \theta\right)\left(\theta \Gamma_{m_{1} \cdots m_{7}} \theta\right)\right\rangle \\
& =: \lambda^{B_{1}} \cdots \lambda^{B_{7}} \theta^{A_{1}} \cdots \theta^{A_{9}} \mathcal{T}_{\left(\left(B_{1} \cdots B_{7}\right)\right)\left[A_{1} \cdots A_{9}\right]}
\end{aligned}
$$

and write

$$
\left[d^{23} \lambda\right]^{\left[\alpha_{1} \cdots \alpha_{23}\right]}:=d \lambda^{\alpha_{1}} \wedge \cdots \wedge d \lambda^{\alpha_{23}}=[\mathcal{D} \lambda]_{+16}\left(\epsilon_{32} \mathcal{T}\right)_{\left(\left(\beta_{1} \cdots \beta_{7}\right)\right)}^{\left[\alpha_{1} \cdots \alpha_{23}\right]} \lambda^{\beta_{1}} \cdots \lambda^{\beta_{7}}
$$

with $\epsilon_{32}$ the totally antisymmetric tensor $\epsilon^{\alpha_{1} \cdots \alpha_{32}}$ and $[\mathcal{D} \lambda]_{+16}$ as scalar measure factor with ghost-charge +16 .

The inverse tensor $\mathcal{T}^{-1}$ is given by

$$
\begin{aligned}
\left(\mathcal{T}^{-1}\right)_{\left[\rho_{1} \cdots \rho_{9}\right]}^{\left(\left(\alpha_{1} \cdots \alpha_{7}\right)\right)} & =\left\langle\lambda^{\alpha_{1}} \cdots \lambda^{\alpha_{7}} \theta_{\rho_{1}} \cdots \theta_{\rho_{9}}\right\rangle \\
& =\prod_{\beta_{1} \cdots \beta_{7}}^{\left(\left(\alpha_{1} \cdots \alpha_{7}\right)\right)}\left(\Gamma^{M_{1}}\right)^{\beta_{1}}{ }_{\left[\rho_{1}\right.} \cdots\left(\Gamma^{M_{7}}\right)^{\beta_{7}}{ }_{\left.\rho_{7}\right]}\left(\Gamma_{M_{1} \cdots M_{7}}\right)_{\left.\rho_{8} \rho_{9}\right]}
\end{aligned}
$$


where $\Pi_{\beta_{1} \cdots \beta_{7}}^{\left(\left(\alpha_{1} \cdots \alpha_{7}\right)\right)}$ is the projector on symmetric $\Gamma_{M}$-traceless 7 -tensors:

$$
\begin{aligned}
\Pi_{\beta_{1} \cdots \beta_{7}}^{\left(\left(\alpha_{1} \cdots \alpha_{7}\right)\right)} & =\delta_{\beta_{1}}^{\left(\alpha_{1}\right.} \cdots \delta_{\beta_{7}}^{\left.\alpha_{7}\right)}-\frac{21}{74}\left(\Gamma^{M_{1}}\right)^{\left(\alpha_{1} \alpha_{2}\right.} \delta_{\left(\beta_{1}\right.}^{\alpha_{3}} \cdots \delta_{\beta_{5}}^{\left.\alpha_{7}\right)}\left(\Gamma_{M_{1}}\right)_{\left.\beta_{6} \beta_{7}\right)} \\
& \left.+\frac{21}{592}\left(\Gamma^{M_{1}}\right)^{\left(\alpha_{1} \alpha_{2}\right.}\left(\Gamma^{M_{2}}\right)^{\alpha_{3} \alpha_{4}} \delta^{\alpha_{5}}{ }_{\left(\beta_{1}\right.} \cdots \delta_{\beta_{3}}^{\left.\alpha_{7}\right)}\left(\Gamma_{M_{2}}\right)_{\left.\beta_{4} \beta_{5}\right)}\left(\Gamma_{M_{1}}\right)_{\left.\beta_{6} \beta_{7}\right)}\right) \\
& \left.-\frac{21}{20128}\left(\Gamma^{M_{1}}\right)^{\left(\alpha_{1} \alpha_{2}\right.} \cdots\left(\Gamma^{M_{3}}\right)^{\alpha_{5} \alpha_{6}} \delta^{\left.\alpha_{7}\right)}{ }_{\left(\beta_{1}\right.}\left(\Gamma_{M_{3}}\right)_{\beta_{2} \beta_{3}} \cdots\left(\Gamma_{M_{1}}\right)_{\beta_{6} \beta_{7}}\right) .
\end{aligned}
$$

Before concluding this subsection we make a few remarks to stress the differences between the eleven-dimensional case studied in the present paper and the ten-dimensional setup.

We can parametrize the pure spinor $\lambda^{A}$ by first splitting the $\mathbf{3 2}$ of $\mathrm{SO}(11)$ as $\mathbf{1 6} \oplus \mathbf{1 6}$ of $\mathrm{SO}(10)$, i.e. $\lambda^{A}=\left(\lambda_{L}^{\alpha}, \lambda_{R}^{\alpha}\right)$, and then decomposing each $\mathrm{SO}(10)$ spinor into $\mathrm{U}(5)$ representations $\mathbf{1} \oplus \mathbf{5} \oplus \overline{\mathbf{1 0}}$ and $\overline{\mathbf{1}} \oplus \overline{\mathbf{5}} \oplus \mathbf{1 0}$ as

$$
\lambda_{L}^{\alpha}=\left(\lambda_{+}, \lambda_{a}, \lambda^{a b}\right), \quad \lambda_{R}^{\alpha}=\left(\lambda_{-}, \lambda^{a}, \lambda_{a b}\right), \quad a=1, \cdots, 5
$$

In this language the pure spinor constraint reads

$$
\begin{aligned}
\lambda_{+} \lambda_{-}+\lambda_{a} \lambda^{a}+\frac{1}{2} \lambda_{a b} \lambda^{a b} & =0 \\
\lambda_{+} \lambda_{a}+\frac{1}{8} \epsilon_{a b c d e} \lambda^{b c} \lambda^{d e} & =-\lambda^{b} \lambda_{a b} \\
\lambda_{-} \lambda^{a}+\frac{1}{8} \epsilon^{a b c d e} \lambda_{b c} \lambda_{d e} & =-\lambda_{b} \lambda^{a b}
\end{aligned}
$$

where the first equation comes from $\lambda \Gamma^{11} \lambda=0$ and the other two from $\lambda_{L} \Gamma^{m} \lambda_{L}+$ $\lambda_{R} \Gamma^{m} \lambda_{R}=0$. We solve these equations as

$$
\begin{aligned}
& 5 ! \lambda^{[a} \lambda^{b c} \lambda^{d e]}=8 \epsilon^{a b c d e} \lambda_{+}\left(\lambda_{+} \lambda_{-}+\frac{1}{2} \lambda_{a b} \lambda^{a b}\right) \\
& 5 ! \lambda_{[a} \lambda_{b c} \lambda_{d e]}=8 \epsilon_{a b c d e} \lambda_{-}\left(\lambda_{+} \lambda_{-}+\frac{1}{2} \lambda_{a b} \lambda^{a b}\right) .
\end{aligned}
$$

This determines completely all components of $\lambda_{a}$ and $\lambda^{a}$. The solution is very similar to the one of the ten-dimensional pure spinor constraint, where the $\mathbf{5}$ is solved in terms of the $\overline{\mathbf{1 0}}$ of $\mathrm{U}(5)$ as $8 \lambda_{+} \lambda_{a}=-\epsilon_{\text {abcde }} \lambda^{b c} \lambda^{\text {de }}$ (see [10). But there is a crucial difference since the 5 of $\lambda_{L}$ is solved in terms of the $\mathbf{1}$ and $\mathbf{1 0}$ of $\lambda_{L}$ and $\lambda_{R}$. This gives a solution with $2 \times(1+10)=22$ complex parameters. The $\mathrm{SU}(5)$ decomposition of an $\mathrm{SO}(11)$ pure spinor has an extra complex parameter $\rho$, which leaves equations (4.8) and (4.9) invariant under the rescaling

$$
\left(\lambda_{L}, \lambda_{R}\right) \rightarrow\left(\rho \lambda_{L}, \rho^{-1} \lambda_{R}\right)
$$


This symmetry is related to the constraint $\lambda \Gamma^{11} \lambda=0$ (i.e. $\lambda_{L, \alpha} \lambda_{R}^{\alpha}=0$; this condition can be solved by a generic choice of $\lambda_{\alpha, L}$ and $\lambda_{R}^{\alpha}$ up to a scale gauge symmetry), which states that an eleven dimensional pure spinor is not just the square of two ten dimensional pure spinors. It is now clear that the measure on $\lambda^{A}$ can also be written in the following form:

$$
d^{23} \lambda^{A}=d \rho \wedge\left(d \lambda_{+} \wedge d \lambda^{a b}\right) \wedge\left(d \lambda_{-} \wedge d \lambda_{a b}\right)
$$

\subsection{Tree-level action from scattering amplitudes}

In this subsection we will reproduce the effective action for eleven dimensional supergravity up to four-fermi terms from tree-level amplitude computations. The supergravity action up to this order in the fermion fields reads 15

$$
S=\frac{1}{2 \ell_{P}^{9}} \int d^{11} x\left[e \mathcal{R}+e \bar{\Psi}_{M} \Gamma^{M N P} \hat{D}_{N} \Psi_{P}+\frac{1}{2} G_{4} \wedge * G_{4}+\frac{1}{6} C_{3} \wedge G_{4} \wedge G_{4}\right]
$$

where $\hat{D}_{M}=\partial_{M}+\frac{1}{4} \omega_{M}^{r s} \Gamma^{r s}+\mathcal{T}_{M}{ }^{r_{1} \cdots r_{4}} G_{r_{1} \cdots r_{4}}$ is the supercovariant derivative (again up to fermion bilinears), involving the $G_{4}$ field strength. The tensor $\mathcal{T}_{M}{ }^{r_{1} \cdots r_{4}}$ is defined in (3.7).

The zero momentum cohomology of $Q$ contains a non zero $\lambda$-ghost number 3 vertex operator $U^{(3)}$ whose components are comprised of the supergravity fields and their derivatives and a $\lambda$-ghost number 4 vertex operator $U^{(4)}$ containing the antifields. The relevant components of $U^{(3)}$ are 10$]$ :

$$
\begin{aligned}
U_{C_{3}}^{(3)} & =\left(\lambda \Gamma^{M} \theta\right)\left(\lambda \Gamma^{N} \theta\right)\left(\lambda \Gamma^{K} \theta\right) C_{M N K} e^{i k \cdot X} \\
U_{g}^{(3)} & =\left(\lambda \Gamma^{(M} \theta\right)\left(\lambda \Gamma^{N) K} \theta\right)\left(\lambda \Gamma_{K} \theta\right) g_{M N} e^{i k \cdot X} \\
U_{\Psi}^{(3)} & =\left(\lambda \Gamma^{M} \theta\right)\left[\left(\lambda \Gamma^{N} \theta\right)\left(\lambda \Gamma^{P} \theta\right)\left(\theta \Gamma^{N P} \Psi_{M}\right)\right. \\
& \left.-\left(\lambda \Gamma^{N P} \theta\right)\left(\lambda \Gamma^{N} \theta\right)\left(\theta \Gamma^{P} \Psi_{M}\right)\right] e^{i k \cdot X} .
\end{aligned}
$$

Derivatives of the supergravity fields appear at higher orders in the $\theta$ expansion of $U^{(3)}$. The $U^{(4)}$ vertex operator has a similar expression in terms of the antifields. However, it does not enter the definition of tree-level superparticle amplitudes as it is intrinsically related to the supermembrane. We will comment more on $U^{(4)}$ below.

For performing amplitude computations we also need the part of the $U^{(1)}$ vertex operator (3.3) which contains the gravitino and the 3-form field strength:

$$
\begin{aligned}
U_{\Psi}^{(1)} & =\left(\lambda \Gamma_{N} \theta\right)\left(\theta \Gamma_{M} \Psi^{N}\right) P^{M} e^{i k \cdot X} \\
U_{G_{4}}^{(1)} & =\left(\lambda \Gamma_{N} \theta\right)\left(\theta \mathcal{U}^{N \mid r_{1} \cdots r_{4} M} \theta\right) G_{r_{1} \cdots r_{4}} P^{M} e^{i k \cdot X} .
\end{aligned}
$$

The tensor $\mathcal{U}^{N \mid r_{1} \cdots r_{4} M}$ is defined in (3.8). Because $U_{C_{3}, g, \Psi}^{(3)}$ are physical vertex operators, $\left\{Q, U^{(3)}\right\}=0$ implies the equations of motion of eleven dimensional supergravity [10]. 
This means that all terms containing two fields in the action (4.12) are derivable from the amplitude $\left\langle U^{(3)}|Q| U^{(3)}\right\rangle$. The cubic term in the supergravity action (4.12) is obtained from the amplitude $\left\langle U^{(3)} U^{(1)} U^{(3)}\right\rangle$.

For instance, we first consider the amplitude given by the insertion of two $U^{(3)}$ vertex operators for the $C_{3}$-field at positions $\tau_{1}$ and $\tau_{2}$ and a $C_{3}$ vertex operator $U^{(1)}$ at position $\tau_{3}:$

$$
\begin{aligned}
& \left\langle U_{C_{3}}^{(3)}\left(\tau_{1}\right) U_{C_{3}}^{(3)}\left(\tau_{2}\right) U_{G_{4}}^{(1)}\left(\tau_{3}\right)\right\rangle=C_{M_{1} \cdots M_{3}} C_{N_{1} \cdots N_{3}} G_{r_{1} \cdots r_{4}} \\
\times & \left\langle\left(\lambda \Gamma^{M_{1}} \theta\right) \cdots\left(\lambda \Gamma^{M_{3}} \theta\right)\left(\lambda \Gamma^{N_{1}} \theta\right) \cdots\left(\lambda \Gamma^{N_{3}} \theta\right)\left(\lambda \Gamma^{N} \theta\right)\left(\theta \mathcal{U}^{N \mid r_{1} \cdots r_{4} M} \theta\right) P^{M} \prod_{i=1}^{3} e^{i k^{(i)} \cdot X}\right\rangle .
\end{aligned}
$$

Using that on shell $\left(k^{(i)}\right)^{2}=k^{(i)} \cdot k^{(j)}=0$ for $i, j=1,2,3, P^{M}=\dot{X}^{M}$ and also the tree-level correlator of the world-line formalism [13]:

$$
\left\langle X^{M}\left(\tau_{1}\right) X^{N}\left(\tau_{2}\right)\right\rangle_{\text {tree }}=\eta^{M N}\left(\frac{\left|\tau_{1}-\tau_{2}\right|}{2}+a+b \tau_{2}\right),
$$

where $a$ and $b$ are arbitrary constants not entering in any physical amplitude, the contraction with the plane-wave from a $U^{(3)}$ vertex operator gives $\left\langle P^{M}\left(\tau_{3}\right) \exp \left(i k^{(i)} \cdot X\left(\tau_{i}\right)\right)\right\rangle=$ $i\left(k^{(i)}\right)^{M} \operatorname{sign}\left(\tau_{13}\right)$. So assuming that $\tau_{1}<\tau_{3}<\tau_{2}$ we obtain:

$$
\begin{aligned}
& \left\langle U_{C_{3}}^{(3)}\left(\tau_{1}\right) U_{C_{3}}^{(3)}\left(\tau_{2}\right) U_{G_{4}}^{(1)}\left(\tau_{3}\right)\right\rangle=\frac{1}{288} C_{M_{1} \cdots M_{3}} C_{M_{4} \cdots M_{6}} G_{r_{1} \cdots r_{4}}\left[\left(k^{(2)}\right)_{L}-\left(k^{(1)}\right)_{L}\right] \\
\times & \left\langle\left(\lambda \Gamma^{M_{1}} \theta\right) \cdots\left(\lambda \Gamma^{M_{7}} \theta\right)\left[5 \eta^{M_{7}[L}\left(\theta \Gamma^{\left.r_{1} \cdots r_{4}\right]} \theta\right)+8\left(\theta \Gamma^{M_{7}\left[r_{1} \cdots r_{3}\right.} \theta\right) \eta^{\left.r_{4}\right] L}\right]\right\rangle .
\end{aligned}
$$

Because of the relation

$$
\left\langle\left(\lambda \Gamma^{M_{1}} \theta\right) \cdots\left(\lambda \Gamma^{M_{7}} \theta\right)\left(\theta \Gamma^{M_{8} \cdots M_{11}} \theta\right)\right\rangle \propto \epsilon_{11}^{M_{1} \cdots M_{11}},
$$

with the proportionality constant fixed by the normalization (2.15), this amplitude reproduces the Chern-Simons coupling of the supergravity action (4.12)

$$
\left\langle U_{C_{3}}^{(3)} U_{C_{3}}^{(3)} U_{G_{4}}^{(1)}\right\rangle=\epsilon_{11}^{M_{1} \cdots M_{11}} C_{M_{1} \cdots M_{3}} G_{M_{4} \cdots M_{7}} G_{M_{8} \cdots M_{11}} .
$$

The other coupling $\Psi_{M} \Gamma^{M N P} \mathcal{T}_{N} \cdot G_{4} \Psi_{P}$ from the supercovariant derivative can be derived in the same way from the amplitude $\left\langle U_{C_{3}}^{(3)} U_{\Psi}^{(3)} U_{\Psi}^{(1)}\right\rangle$, which is the only one compatible with the tree level normalisation $\left\langle\lambda^{7} \theta^{9}\right\rangle$.

We can therefore summarize the two-derivative non-linear eleven dimensional supergravity action in the following way:

$$
S_{11 d}=\left\langle U^{(3)} Q U^{(3)}\right\rangle+\left\langle U^{(3)}\left[U^{(1)}, U^{(3)}\right]\right\rangle,
$$


where $\left[U^{(1)}, U^{(3)}\right]$ means using the canonical bracket for the on-shell fields $P^{M}$ and $X_{N}$ appearing in $U^{(1)}$ and $U^{(3)}$. The symbol $\langle\cdots\rangle$ denotes the correlator for zero modes using the measure (2.15). The action (4.18) varies under the gauge transformation $\delta U^{(3)}=\{Q+$ $\left.U^{(1)}, \Omega^{(2)}\right\}$ into $\left\langle U^{(3)}\left[U^{(1)},\left\{U^{(1)}, \Omega^{(2)}\right\}\right]\right\rangle$. The first term in the transformation, $\left\{Q, \Omega^{(2)}\right\}$, generates gauge symmetries on the physical fields, whereas the second term, $\left\{U_{\Phi}^{(1)}, \Omega^{(2)}\right\}=$ $\delta U_{\delta \Phi}^{(3)}$, generates supersymmetry transformations $\delta \Phi$ on the physical fields. The nonzero variation of the action is due to neglecting ${ }^{7}$ the four fermion terms in (4.18) necessary for supersymmetry invariance of $(4.12){ }^{8}$

\section{Definition of the one-loop amplitude}

Using a world-line path integral formulation of a loop amplitude, it is necessary to integrate over the Schwinger parameters, the loop momenta, the 32 fermionic variables $\theta^{A}$, the 32 supersymmetric Green-Schwarz constraints $d_{A}$ (representing the conjugate momenta $\left.p_{A}\right)$, the pure spinors $\lambda^{A}$ and their conjugate ghosts $w_{A}$. As explained in [12] it is as well necessary to insert picture raising operators $Z_{B}$ and $Z_{J}$

$$
\begin{aligned}
Z_{B} & =B_{M N}\left(\lambda \Gamma^{M N} d\right) \delta\left(B_{M N} N^{M N}\right) \\
Z_{J} & =\lambda d \delta(J)
\end{aligned}
$$

and picture lowering operators

$$
Y_{C}=C_{A} \theta^{A} \delta\left(C_{A} \lambda^{A}\right)
$$

for absorbing the zero modes of $\lambda^{A}$ and $w_{A}$. As usual, at one loop translation invariance requires to have at least one unintegrated vertex operator. Summarizing, the amplitude is defined as:

$$
\mathcal{A}=\int \frac{d \tau}{\tau}\left\langle b_{B} Z_{J} \prod_{P=2}^{22} Z_{B} \prod_{I=1}^{23} Y_{C_{I}} U^{(1)}\left(\tau_{1}\right) \prod_{T=2}^{N} \int_{0}^{\tau} d \tau^{T} \mathcal{V}\left(\tau^{T}\right)\right\rangle
$$

where $U^{(1)}$ is the unintegrated vertex operator defined as $\{Q, \mathcal{V}(\tau)\}=: \partial_{\tau} U^{(1)}$. Notice that the vertex operator $U^{(1)}$ has ghost-number 1 and contains $d_{A}$ terms (see (3.15)). ${ }^{9}$

\footnotetext{
${ }^{7}$ The reason we have not written out these terms is only aesthetical, not conceptual. They arise as contact terms of the four-point amplitude containing an integrated vertex operator.

${ }^{8}$ Recall that they are taken care of by completing the spin connection and four-form with fermion bilinears to get a supercovariant derivative acting on the fermions 15 .

${ }^{9}$ We already pointed out that the tree-level amplitudes for the superparticle do not involve the ghost number 4 vertex operator $U^{(4)}$. By unitary arguments, this vertex does not enter the definition of superparticle loop amplitudes either.
} 
Before specifying the measure of integration over the $\lambda^{\alpha}$ and $w_{\alpha}$, we first stress that this amplitude cannot be just a trivial lift of the one-loop superstring prescription of Berkovits, since the number of components of $\lambda^{\alpha}$ is 23 which is with one more than for the left/right doublet of the ten-dimensional pure spinors $\left(\lambda_{L}^{\alpha}, \lambda_{R}^{\alpha}\right)$.

Let us also check that the zero mode counting for $\theta^{A}, d_{A}$, and $\lambda_{A}$ works:

$\triangleright$ We start by enumerating the $d_{\alpha}$ zero modes. At one-loop 32 have to be soaken up. Clearly one gets 21 from $Z_{B}, 1$ from $Z_{J}, 1$ from $U^{(1)}$ and at most $2(N-1)-M$ from the integrated vertex operators $\mathcal{V}$ (notice that the $\mathcal{V}$ 's depend on the quadratic combinations: $d d, d N$ or $N N$ ), where $M$ is the number of $N^{N M}$ zero modes. So far, the counting of $d_{\alpha}$ zero modes gives $32-21-1-1+2-2 N+M=11-2 N+M$. Therefore for the four-point amplitude, $N=4$, we have to extract $4 d$ 's from $b_{B}$. Since the latter come together with a $\delta^{\prime}\left(B_{M N} N^{M N}\right)$, which counts as $-1 N^{N M}$ zero mode, we also have to pick $M=1$ to saturate it. Morever, the above counting shows that the first non-vanishing one-loop amplitude is with four external states.

$\triangleright$ Now we count the $\theta$ zero modes. There are 32 of them to integrate over; the picture lowering operators $Y_{C}$ contain 23 so 9 have to arise from the vertex operators. For the case $N=4$ and $M=1$, we already get $5 \theta$ 's from the integrated vertex operators. So $U^{(1)}$ has to contribute $4 \theta$ zero modes.

$\triangleright$ The $\lambda$-ghost counting goes as follows: We get +21 zero modes from $Z_{B},+1$ from $Z_{J},+1$ from $U^{(1)}$ and -23 from $Y_{C}$, for a total of

$$
21+1-23+1=0 \text {. }
$$

\subsection{Measure for the conjugate momentum $w_{A}$}

The constraints (4.3) allow the gauge symmetry $\delta_{\Lambda} w_{A}=\Lambda_{M} \Gamma_{A B}^{M} \lambda^{B}$. The only bilinears, $\left(w \Gamma^{M_{1} \cdots M_{r}} \lambda\right)$, invariant under it are the Lorentz generators $N^{M N}=\left(w \Gamma^{M N} \lambda\right) / 2$ and the ghost-number operator $J=(w \lambda)$. The reason is that the variation $\delta_{\Lambda}\left(w \Gamma^{M_{1} \cdots M_{r}} \lambda\right)=$ $\left(\delta_{\Lambda} w \Gamma^{M_{1} \cdots M_{r}} \lambda\right)=\Lambda_{P}\left(\lambda \Gamma^{P M_{1} \cdots M_{r}} \lambda\right)+r \Lambda^{\left[M_{1}\right.}\left(\lambda \Gamma^{\left.M_{2} \cdots M_{r}\right]} \lambda\right)$ vanishes only for $r=0$ and $r=2$ because of the commuting nature of $\lambda^{A}$. Generally the vertex operators are also invariant under $\delta_{\Lambda}$ and depend on $N^{[M N]}:=\frac{1}{2}\left(w \Gamma^{M N} \lambda\right)$ and $J=(w \lambda)$. To construct the measure for integration over $w_{A}$ we use the change of variables

$$
32 w_{A} \lambda^{B}=\delta_{A}^{B} J+\frac{1}{2 !}\left(\Gamma_{M N}\right)_{A}{ }^{B} N^{M N}
$$

and expand $\wedge^{23} d w_{A}$ as

$$
\left(\wedge^{23} d w_{A}\right) \lambda^{B_{1}} \cdots \lambda^{B_{23}}=\mathcal{T}_{1}^{m_{1} n_{1} \cdots m_{22} n_{22}}\left(\wedge^{22} d N^{\left[m_{i} n_{i}\right]} \wedge d J\right)+\mathcal{T}_{2}^{m_{1} n_{1} \cdots m_{23} n_{23}}\left(\wedge^{23} d N^{\left[m_{i} n_{i}\right]}\right) .
$$


Doing so we have obtained two contributions to the measure. The tensors $\mathcal{T}_{I=1,2}^{m_{1} \cdots}$ will be determined later.

The measure factors in (5.7) satisfy respectively

$$
\begin{aligned}
\left(\lambda \Gamma_{m_{1}}\right)_{A}\left(\wedge^{22} d N^{\left[m_{i} n_{i}\right]} \wedge d J\right) & =0 \\
\left(\lambda \Gamma_{m_{1}}\right)_{A}\left(\wedge^{23} d N^{\left[m_{i} n_{i}\right]}\right) & =\left(\lambda \Gamma^{n_{1}}\right)_{A}\left(\wedge^{22} d N^{\left[m_{i} n_{i}\right]} \wedge d J\right) .
\end{aligned}
$$

The first equation follows from the relation

$$
N^{M N} \lambda^{A}\left(\Gamma_{M}\right)_{A B}-\frac{1}{2} J \lambda^{A}\left(\Gamma^{N}\right)_{A B}=0,
$$

valid at the classical level. The second one can be checked directly by using the Fierz identities (A.4), (A.6), (A.7) and (A.8). Equation (5.9) shows that the second contribution in (5.7) is not independant of the first one. Hence there is a single measure factor for $\wedge^{23} d w_{A}$ compatible with the pure spinor constraint (4.3).

Counting the number of available Lorentz indices and having at most $23 \lambda^{A}$ we find that the measure can be decomposed in the following two products of $\lambda$-bilinears (see Appendix $\mathrm{A}$ for details about Fierz identities in eleven dimensions): ${ }^{10}$

$$
\wedge^{22} d N^{\left[m_{i} n_{i}\right]} \wedge d J=[\mathcal{D} w]_{-16}\left(\lambda \Gamma_{[5]} \lambda\right)^{4}\left(\lambda \Gamma_{[6]} \lambda\right)^{4}+[\mathcal{D} w]_{-20}\left(\lambda \Gamma_{[5]} \lambda\right)^{8}\left(\lambda \Gamma_{[2]} \lambda\right)^{2},
$$

where $[\mathcal{D} N]_{g=-16,-20}$ are scalar measure factors of ghost-number -16 and -20 . The constraint (5.8) and the identities in Appendix A forbid the ghost-number -20 term, but allow the other one with ghost-number -16 :

$$
\begin{aligned}
\wedge^{22} d N^{\left[m_{i} n_{i}\right]} \wedge d J & =[\mathcal{D} w]_{-16} \times \\
& \times\left[\left(\lambda \Gamma^{m_{1} n_{1} m_{2} m_{3} m_{4}} \lambda\right)\left(\lambda \Gamma^{m_{5} n_{5} n_{2} m_{6} m_{7}} \lambda\right)\left(\lambda \Gamma^{m_{8} n_{8} n_{3} n_{6} m_{9}} \lambda\right)\left(\lambda \Gamma^{m_{10} n_{10} n_{4} n_{7} n_{9}} \lambda\right)\right. \\
& \times\left(\lambda \Gamma^{m_{11} n_{11} m_{12} m_{13} m_{14} m_{21}} \lambda\right)\left(\lambda \Gamma^{m_{15} n_{15} n_{12} m_{16} m_{17} n_{21}} \lambda\right)\left(\lambda \Gamma^{m_{18} n_{18} n_{13} n_{16} m_{19} m_{22}} \lambda\right) \\
& \left.\times\left(\lambda \Gamma^{m_{20} n_{20} n_{14} n_{17} n_{19} n_{22}} \lambda\right)+\text { perms }\right] .
\end{aligned}
$$

Remarking that $\left(\lambda \Gamma_{[6]} \lambda\right)=\epsilon_{11}\left(\lambda \Gamma_{[5]} \lambda\right)$ we can interpret this product as the left-movers times the right-movers measure factor constructed for the superstring in [12]. For later use, we also note that the Ward identity (5.9) allows an alternative form for the measure factor:

$$
\begin{aligned}
\wedge^{23} d N^{\left[m_{i} n_{i}\right]} & =[\mathcal{D} w]_{-16} \times\left[\left(\lambda \Gamma^{m_{1} n_{1} m_{2} m_{3} m_{4}} \lambda\right)\left(\lambda \Gamma^{m_{5} n_{5} n_{2} m_{6} m_{7}} \lambda\right)\right. \\
& \times\left(\lambda \Gamma^{m_{8} n_{8} n_{3} n_{6} m_{9} m_{23}} \lambda\right)\left(\lambda \Gamma^{m_{10} n_{10} n_{4} n_{7} n_{9} n_{23}} \lambda\right) \\
& \times\left(\lambda \Gamma^{m_{11} n_{11} m_{12} m_{13} m_{14} m_{21}} \lambda\right)\left(\lambda \Gamma^{m_{15} n_{15} n_{12} m_{16} m_{17} n_{21}} \lambda\right)\left(\lambda \Gamma^{m_{18} n_{18} n_{13} n_{16} m_{19} m_{22}} \lambda\right) \\
& \left.\times\left(\lambda \Gamma^{m_{20} n_{20} n_{14} n_{17} n_{19} n_{22}} \lambda\right)+\text { perms }\right] .
\end{aligned}
$$

\footnotetext{
${ }^{10}$ In the following the antisymmetric product of $n$ Gamma-matrices $\Gamma_{M_{1} \cdots M_{n}}$ is abreviated as $\Gamma_{[n]}$.
} 


\subsection{A different representation of the $b_{B}$ ghost}

An important peculiarity of Berkovits' pure spinor cohomology is that the energy momentum tensor $T=P^{2} / 2$ is not $Q$-exact. One can define a (bi-local) ghost $\widetilde{b}_{B}$ by requiring that 12

$$
\left\{Q, \widetilde{b}_{B}(u, z)\right\}=Z_{B}(u) T(z),
$$

where

$$
Z_{B}=B_{M N}\left(\lambda \Gamma^{M N} d\right) \delta\left(B_{M N} N^{M N}\right) .
$$

Notice that, since we are considering the superparticle, all the fields entering $Z_{B}$ are constant and therefore we do not need to specify the position. The same is also true for the picture raising operator $Y_{C_{I}}$ defined by $Y_{C}=C_{A} \theta^{A} \delta\left(C_{A} \lambda^{A}\right)$. Nevertheless, we will keep writing the position argument for generality as the manipulations we do in the rest of this subsection are valid for string theory as well.

Instead of working with a bi-local ghost $\widetilde{b}_{B}(u, z)$ we consider a local one $\widetilde{b}_{B}(u)$ defined up to exact terms as

$$
\widetilde{b}_{B}(u, z)=\widetilde{b}_{B}(u)+\left\{Q, \int_{u}^{z} \Omega\right\}
$$

where $\Omega(u, v)$ is a bi-local superfield. In addition, by recalling that $\partial \Theta(x)=\delta(x)$, we find an alternative to the solution of [12]:

$$
\left\{Q, \widetilde{b}_{B}(u)\right\}=B_{M N} \lambda \Gamma^{M N} d \delta\left(B_{M N} N^{M N}\right) T(u)=\left\{Q, \Theta\left(B_{M N} N^{M N}\right) T(u)\right\}
$$

where we have used the BRST invariance of $T(u)$. Hence

$$
\widetilde{b}_{B}(u)=T(u) \Theta\left(B_{M N} N^{M N}\right)+\{Q, \Omega\},
$$

where the second BRST-exact term is clearly unimportant. The same representation applies to the left and right $b_{B}^{L / R}$ ghosts for type II superstrings.

Finally notice that, using an integral representation for the $\Theta$-function, the insertion of the $b_{B}^{L / R}$ in the one-loop superstring amplitude, can be rewritten as

$$
\int \mu(z, \bar{z}) b_{B}^{L} b_{B}^{R}=\int \mu(z, \bar{z}) \int d t d s \frac{e^{i B_{m n}\left[(t+s)\left(N^{L, m n}+N^{R, m n}\right)+(t-s)\left(N^{L, m n}-N^{R, m n}\right)\right]}}{(t+i \epsilon)(s+i \epsilon)} .
$$

Clearly, the second term in the exponent, that involves the difference of the Lorentz generators, imposes the level matching condition. 


\subsubsection{Action of the $b_{B}$ ghost on vertex operators}

Let us consider the action of the $b_{B}$ ghost on a vertex operator $U^{(q+1)}(\tau)$ with ghostnumber $(q+1)$ :

$$
\widetilde{b}_{B}(u) U^{(q+1)}(\tau)=\Theta(B \cdot N) T(u) U^{(q+1)}(\tau) .
$$

Using the fact that for $q \geq 0, U^{(q+1)}=\lambda^{A} \Phi_{A}^{(q)}$ and that $\left\{Q, G^{A}\right\}=\frac{P^{2}}{2} \lambda^{A}$, where $G^{A}=$ $\Gamma_{M}^{A B} P^{M} d_{B}$, we can rewrite (5.19) as

$$
\begin{aligned}
& \Theta(B \cdot N) T(u) U^{(q+1)}(\tau)=\left\{Q, G^{A}\right\} \Phi_{A}^{(q)} \Theta(B \cdot N) \\
= & \left\{Q, G^{A} \Phi_{A}^{(q)} \Theta(B \cdot N)\right\}-G^{A} \lambda^{B} D_{B} \Phi_{A}^{(q)} \Theta(B \cdot N)-G^{A} \Phi_{A}^{(q)} Z_{B},
\end{aligned}
$$

Now we can use that $\left\{Q, H^{A B}\right\}=G^{A} \lambda^{B}+g^{((A B))}$, where $H^{A B}$ is given by ${ }^{11}$

$$
\begin{aligned}
H^{A B} & =\frac{1}{64}\left[C^{A B}\left(d_{C} C^{C D} d_{D}\right)+\Gamma_{M N P Q}^{A B}\left(d \Gamma^{M N P Q} d\right)\right. \\
& \left.+\Gamma_{M N P}^{A B}\left(d \Gamma^{M N P} d+2 N^{M N} P^{P}\right)+2 \Gamma_{M}^{A B}\left(N^{M R} P_{R}+J P^{M}\right)\right]
\end{aligned}
$$

and $g^{((A B))}$ is traceless and symmetric, to reabsorb part of the vertex into $Q$-exact terms:

$$
\begin{aligned}
\Theta\left(B_{M N} N^{M N}\right) T(u) U^{(q+1)}(\tau) & =-\left[G^{A} \Phi_{A}^{(q)}-H^{A B} D_{A} \Phi_{B}^{(q)}\right] Z_{B}+\{Q, \Omega\} \\
& +\left[H^{A B} \lambda^{C} D_{C} D_{B} \Phi_{A}^{(q)}+g^{((A B))} D_{A} \Phi_{B}^{(q)}\right] \Theta(B \cdot N)
\end{aligned}
$$

${ }^{11}$ It is easy to see that the structure of $H^{A B}$ is the following:

$$
H^{A B}=X^{A B[C D]} d_{C} d_{D}+Y_{M N P}^{A B} P^{M} N^{N P}+Z_{M}^{A B} P^{M} J
$$

where

$$
\begin{aligned}
X^{A B[C D]} & =\mathbf{x} C^{A B} C^{[C D]}+\mathbf{x}^{\prime} \Gamma_{M N P}^{A B}\left(\Gamma^{M N P}\right)^{[C D]}+\mathbf{x}^{\prime \prime} \Gamma_{M N P Q}^{A B}\left(\Gamma^{M N P Q}\right)^{[C D]} \\
Y_{M N P}^{A B} & =\mathbf{y} \Gamma_{M N}^{A B}+\mathbf{y}^{\prime} \Gamma_{N}^{A B} \eta_{P M} \\
Z_{M}^{A B} & =z \Gamma_{M}^{A B}
\end{aligned}
$$

and $\mathbf{x}, \mathbf{x}^{\prime}, \mathbf{x}^{\prime \prime}, \mathbf{y}, \mathbf{y}^{\prime}, \mathbf{z}$ are unknown numerical constants. What is more involved is to calculate the numerical coefficients. One can do that by computing $\left\{Q, H^{A B}\right\}-\lambda^{A} G^{B}$. Since this is equal to $g^{((A B))}$, i.e. it is symmetric and traceless, one finds conditions for the unknown constants from the requirement that the trace and antisymmetric part of this expression vanish. It is most easy to extract those conditions by contracting $\left\{Q, H^{A B}\right\}-\lambda^{A} G^{B}$ with $\Gamma_{A B}^{M^{\prime}}, \Gamma_{A B}^{M^{\prime} N^{\prime} P^{\prime}}, \Gamma_{A B}^{M^{\prime} N^{\prime} P^{\prime} Q^{\prime}}$ and $C_{A B}$. Two of the resulting four equations contain two independent structures each and so one obtains six equations which determine uniquely the six constants. 
Then, making use of $\left\{Q, K^{A B C}\right\}=H^{A B} \lambda^{C}+h_{1}^{((A B)) C}+h_{2}^{A((B C))}$, we rewrite the first term on the second line as before:

$$
\begin{aligned}
& \Theta\left(B_{M N} N^{M N}\right) T(u) U^{(q+1)}(\tau)=\{Q, \Omega\} \\
- & {\left[G^{A} \Phi_{A}^{(q)}-H^{A B} D_{A} \Phi_{B}^{(q)}-K^{A B C} D_{C} D_{B} \Phi_{A}^{(q)}\right] Z_{B} } \\
+ & {\left[K^{A B} \lambda^{D} D_{D} D_{C} D_{B} \Phi_{A}^{(q)}+g^{((A B))} D_{A} \Phi_{B}^{(q)}+h_{i}^{A B C} D_{C} D_{B} \Phi_{A}^{(q)}\right] \Theta(B \cdot N) . }
\end{aligned}
$$

Finally, using that $\left\{Q, L^{A B C D}\right\}=\lambda^{A} K^{B C D}+k_{1}^{((A B)) C D}+k_{2}^{A((B C)) D}+k_{3}^{A B((C D))}$, we obtain:

$$
\begin{aligned}
& \Theta\left(B_{M N} N^{M N}\right) T(u) U^{(q+1)}(\tau)=\{Q, \Omega\} \\
- & {\left[G^{A} \Phi_{A}^{(q)}-H^{A B} D_{A} \Phi_{B}^{(q)}-K^{A B C} D_{C} D_{B} \Phi_{A}^{(q)}+L^{A B C D} D_{D} D_{C} D_{B} \Phi_{A}^{(q)}\right] Z_{B} } \\
+ & {\left[g^{((A B))} D_{A} \Phi_{B}^{(q)}+h_{i}^{A B C} D_{C} D_{B} \Phi_{A}^{(q)}+k_{i}^{A B C D} D_{D} D_{C} D_{B} \Phi_{A}^{(q)}\right] \Theta(B \cdot N) . }
\end{aligned}
$$

However, due to $\left\{D_{A}, D_{B}\right\}=P_{M}\left(\Gamma^{M}\right)_{A B}$ and $D_{(A} \Phi_{B)}^{(q)} \sim\left(\Gamma^{M}\right)_{A B} \Phi_{M}^{(q)}$ all tensors appearing in the third line are $\Gamma_{[1]}$-traceless and so the whole expression multiplying $\Theta(B \cdot N)$ vanishes. We can interpret the second line in (5.24) as the vertex operator $U^{(q+1)}$ as written in a different ' $b$-picture':

$$
\begin{aligned}
V_{B}^{(q+1)}(\tau) & =-\left[G^{A} \Phi_{A}^{(q)}-H^{A B} D_{A} \Phi_{B}^{(q)}-K^{A B C} D_{C} D_{B} \Phi_{A}^{(q)}+L^{A B C D} D_{D} D_{C} D_{B} \Phi_{A}^{(q)}\right] Z_{B} \\
& =\hat{V}^{(q)}(\tau) Z_{B} .
\end{aligned}
$$

Note that these manipulations do not change the ghost number, since $Z_{B}$ has ghost number 1 and $\hat{V}^{(q)}$ has ghost number $q$. Comparing with Berkovits' expression for the $b(z)$-ghost in section 4.2 of [12], the vertex operator $\hat{V}^{(q)}$ is a reshuffling of all terms without a derivative on $\delta(B \cdot N)$ whereas all terms with a derivative on the delta-function in [12] are now moved to the $Q$-exact piece. The operators that enter the definition of $\hat{V}^{(0)}$ are of the symbolic form ${ }^{12}$ :

$$
\begin{aligned}
T & =P P \\
G^{A} & =d P \\
H^{A B} & =d d+N P+J P \\
K^{A B C} & =N d+J d \\
L^{A B C D} & =N N+J N+J J .
\end{aligned}
$$

\footnotetext{
${ }^{12}$ We restrict ourselves to the superparticle case and do not specify the constant coefficients, which can be computed as explained in the previous footnote. For more details we refer the reader to section 4 of [12].
} 
The operators $T$ and $G$, which enforce the Virasoro and $\kappa$-symmetry constraints respectively, are already present without the introduction of pure spinors. On the other hand, the operators $H, K$ and $L$ are new since they contain $\lambda^{A}$ and $w_{A} \cdot{ }^{13}$

Using the above manipulations, we can rewrite the one-loop amplitude prescription in the following way:

$$
\mathcal{A}=\int \frac{d \tau}{\tau}\left\langle Z_{J} \prod_{P=1}^{22} Z_{B} \prod_{I=1}^{23} Y_{C_{I}} \hat{V}^{(0)}\left(\tau_{1}\right) \prod_{T=2}^{N} \int d \tau^{T} V\left(\tau^{T}\right)\right\rangle .
$$

The counting of zero modes goes as in Section 5 , especially if one realizes that the expression (5.25) just gives a different distribution of the $\lambda$ 's, $d$ 's and $\theta$ 's. This counting shows that only the second term in $\hat{V}_{B}^{(0)}$, namely $H^{A B} D_{B} \Phi_{A}^{(q)}$, contributes to the four-point amplitude. $^{14}$

Two final remarks: i) Notice that all manipulations are purely algebraic and do not require any supplementary information such as the constraints coming from conformal invariance in string theory. In the latter case, CFT is needed in order to evaluate the contribution of the non-zero modes. But for our purposes the algebraic properties of pure spinors, together with Lorentz covariance, are completely sufficient. To extend the present analysis to the membrane one would need to know the contributions coming from the non-zero mode part of the theory. ii) Even if the prescription given in (5.27) does not seem to be symmetric with respect to the interchange of vertex operators, one can check, using the descent equations for the vertex operators, that it is symmetric.

\section{One loop amplitudes}

As a check on the correctness and feasibility of the prescription for loop calculations in the pure spinor formalism, in this section we compute the effective action terms induced by several one-loop amplitudes: the ten-dimensional $B \wedge X_{8}$, found in [34], the elevendimensional $C \wedge X_{8}$, deduced in [35], and also the supersymmetric $\mathcal{F}^{4}$ in type I.

\footnotetext{
${ }^{13}$ We would like to elaborate on the relation between the structure of the BRST operator and antighost field in the pure spinor approach and the NSR superstring: in the former, $Q$ contains only a single term at zero picture while the antighost $b_{B}$ is a complicated polynomial. In the latter, the BRST charge $Q_{N S R}$ can be decomposed into three pieces, whereas the antighost field $b_{N S R}$ contains only one term. However, there exists a similarity transformation which maps $Q_{N S R}$ into a single nilpotent piece and at the same time maps the field $b_{N S R}$ into a complicated expression that contains exactly the supersymmetry generator and the Virasoro constraints.

${ }^{14}$ In the rest of this paper only the $H^{A B}$ term will be necessary; the other terms will contribute to higher point and/or higher loop amplitudes.
} 


\section{1 $B \wedge X_{8}$ in ten dimensions}

We compute the five-point amplitude $B \wedge t_{8} R^{4}$ both in type IIA and type IIB superstring using the formalism of [12].

For this calculation we will only need the vertex operators for the graviton and the $B$-field in the integrated $V^{(0,0)}$ picture, unintegrated $U^{(1,1)}$ picture, and the mixed pictures $V^{(1,0)}$ and $V^{(0,1)}$. We use the results of [10, 32] and refer to these papers for complete expressions. For our purposes it is enough to take the $B$-field and Riemman curvature to be constant. The relevant vertex operators are

$$
\begin{aligned}
V_{g}^{(0,0)} & =\int d^{2} z R_{m n p q} \mathcal{L}_{L}^{m n} \mathcal{L}_{R}^{p q} e^{i p \cdot X} \\
V_{B}^{(0,0)} & =\int d^{2} z B_{m n} \partial X^{m} \bar{\partial} X^{n} e^{i p \cdot X} \\
U_{g}^{(0,1)} & =\int d z R_{m n p q} \mathcal{L}_{L}^{m n}\left(\lambda_{R} \gamma^{r} \theta_{R}\right)\left(\theta_{R} \gamma_{r}^{p q} \theta_{R}\right) e^{i k \cdot X} \\
U_{B}^{(0,1)} & =\int d z B_{m n} \partial X^{m}\left(\lambda_{R} \gamma^{n} \theta_{R}\right) e^{i k \cdot X} \\
U^{(1,1)} & =\left(g_{m n}+B_{m n}+\eta_{m n} \varphi\right)\left(\lambda_{L} \gamma^{m} \theta_{L}\right)\left(\lambda_{R} \gamma^{m} \theta_{R}\right) e^{i p \cdot X},
\end{aligned}
$$

where $\mathcal{L}_{L, R}^{m n}$ are the Lorentz generators

$$
\mathcal{L}_{L}^{m n}=\frac{1}{2}\left(w_{L} \gamma^{m n} \lambda_{L}\right)+\left(p_{L} \gamma^{m n} \theta_{L}\right)+X^{[m} \partial X^{n]}
$$

with the equivalent expression for the right-movers. Since we only need the zero modes of fields we can, in particular, replace $\left.p_{\alpha}\right|_{0}$ by $\left.d_{\alpha}\right|_{0}+i /\left.2 \partial X_{m}\left(\gamma^{m} \theta_{L}\right)_{\alpha}\right|_{0}$. From now on we drop the subscript 0 , although we will always mean the zero modes of the corresponding fields. So the Lorentz generator becomes

$$
\hat{\mathcal{L}}_{L}^{m n}=\frac{1}{2}\left(w_{L} \gamma^{m n} \lambda_{L}\right)+\left(d_{L} \gamma^{m n} \theta_{L}\right)+\frac{i}{2} \partial X_{l}\left(\theta_{L} \gamma^{l m n} \theta_{L}\right)+X^{[m} \partial X^{n]}
$$

The five-point amplitude with one $B$-field and four gravitons can be written in three different forms depending on how the ghost-charge is distributed among the $B$-field vertex operator and the graviton vertex operator

$$
\begin{aligned}
\mathcal{A}_{1}=\left|\int \mu b Z_{J} \prod_{i=2}^{10} Z_{B} \prod_{i=1}^{11} Y_{C}\right|^{2} U_{B}^{(1,1)}\left(V_{g}^{(0,0)}\right)^{4} \\
\mathcal{A}_{2}=\left|\int \mu b Z_{J} \prod_{i=2}^{10} Z_{B} \prod_{i=1}^{11} Y_{C}\right|^{2} U_{g}^{(1,1)} V_{B}^{(0,0)}\left(V_{g}^{(0,0)}\right)^{3} \\
\mathcal{A}_{3}=\left|\int \mu b Z_{J} \prod_{i=2}^{10} Z_{B} \prod_{i=1}^{11} Y_{C}\right|^{2}\left[U_{B}^{(1,0)} U_{g}^{(0,1)}+U_{B}^{(0,1)} U_{g}^{(1,0)}\right]\left(V_{g}^{(0,0)}\right)^{3}
\end{aligned}
$$


However, the result is independent on the distribution of the ghost number as can be seen in the following way: Write

$$
U^{(1,1)}=\int d^{2} z \partial \bar{\partial} U^{(1,1)}=\left\{Q_{B}^{L}, U^{(0,1)}\right\}=\left\{Q_{B}^{R}, U^{(1,0)}\right\}
$$

and circulate the left (right) BRST charge $Q_{B}^{L}\left(Q_{B}^{R}\right)$ inside the integral. Now, the picture changing operators $Z_{B}$ and $Z_{J}$ are BRST invariant but the $b$ ghost is not, its variation being $\left\{Q_{B}^{L}, b\right\}=Z_{B} T(z)$. Nevertheless, the latter contribution vanishes after integration over the moduli since $T$ gives a total derivative with respect to them.

Despite the equivalence of (6.4)-(6.6), similarly to the NSR formalism, there is a computationally preferred distribution of ghost number, namely the one given by $\mathcal{A}_{3}$. Using the correlator

$$
\left\langle\partial X^{m}(z) \bar{\partial} X^{n}(0)\right\rangle=\pi \alpha^{\prime}\left(\delta^{(2)}(z)-\frac{1}{\tau_{2}}\right) \eta^{m n},
$$

where $\tau_{2}=\operatorname{Im} \tau$, the amplitude reduces to

$$
\begin{aligned}
\mathcal{A}_{3}= & \int \frac{d^{2} \tau}{\tau_{2}^{2}}\left|\int[\mathcal{D} \lambda][\mathcal{D} w] \mathrm{d}^{16} \theta \mathrm{d}^{16} d \int b Z_{J} \prod_{i=2}^{10} Z_{B} \prod_{i=1}^{11} Y_{C}\right|^{2}\left(V_{g}^{(0,0)}\right)^{3} \\
& \times R_{r s t u} B_{[[m n} g_{k l]]}\left[\left(\lambda_{R} \gamma^{n} \theta_{R}\right)\left(\theta_{R} \gamma^{m t u} \theta_{R}\right)\left(\lambda_{L} \gamma^{k} \theta_{L}\right)\left(\theta_{L} \gamma^{l r s} \theta_{L}\right)\right] e^{i k^{(1)} \cdot X} e^{i k^{(2)} \cdot X}
\end{aligned}
$$

with $B_{[[m n} g_{k l]]}=B_{m n} g_{k l}-B_{k l} g_{m n}$. In the 0-ghost-picture vertex operator only the part

$$
\mathcal{M}_{L, R}^{m n}=\frac{1}{2}\left(w_{L, R} \gamma^{m n} \lambda_{L, R}\right)+\left(d_{L, R} \gamma^{m n} \theta_{L, R}\right)
$$

of the Lorentz generator $\hat{\mathcal{L}}_{L}^{m n}$ in (6.3) contributes and after integration over the position of the vertex operator, the leading term in $\alpha^{\prime}$ expansion for $\mathcal{A}_{3}$ reduces to

$$
\mathcal{A}_{3}=\int \frac{d^{2} \tau}{\tau_{2}^{2}} t_{L}^{k l \mid m_{1} \cdots m_{8}} t_{R}^{m n \mid n_{1} \cdots n_{8}} B_{[[m n} g_{k l]]} R_{m_{1} m_{2} n_{1} n_{2}} \cdots R_{m_{7} m_{8} n_{7} n_{8}}
$$

In (6.9) we have defined the left and right tensors ${ }^{15}$

$$
t^{k l \mid m_{1} \cdots m_{8}}=\int[\mathcal{D} \lambda][\mathcal{D} w] b Z_{J} \prod_{i=2}^{10} Z_{B} \prod_{i=1}^{11} Y_{C}\left[\left(\lambda \gamma^{k} \theta\right)\left(\theta \gamma^{l<m_{1} m_{2}} \theta\right) \mathcal{M}^{m_{3} m_{4}} \cdots \mathcal{M}^{m_{7} m_{8}>}\right]
$$

\footnotetext{
${ }^{15}$ Notice that these tensors are elements of the cohomology $H^{(1)}(Q)$ as they arise from an amplitude with one vertex operator in the ghost-number- 1 picture and three vertex operators in the ghost-number-0 picture.
} 
where $\langle\cdots\rangle$ denotes symmetrization over exchanges of the pairs $\left(m_{2 i-1}, m_{2 i}\right) i=$ $1, \ldots, 4$, as a consequence of the many ways of choosing the distribution of the indices when performing the zero mode integration. After integrating over the $\lambda$ 's and $d$ 's as in section 6.3 of [12], one finds that this tensor reduces to

$$
\begin{aligned}
t_{L}^{k l \mid m_{1} \cdots m_{8}}= & \int d^{16} \theta_{L}\left(\epsilon_{16} \mathcal{T}_{L}^{-1}\right)_{\left[\rho_{1} \cdots \rho_{11}\right]}^{\left(\left(\alpha_{1} \cdots \alpha_{3}\right)\right)} \theta_{L}^{\rho_{1}} \cdots \theta_{L}^{\rho_{11}} \\
& \times\left(\gamma_{p_{1} \cdots p_{3}}{ }^{<m_{7} m_{8}}\right)_{\alpha_{2} \alpha_{3}}\left(\gamma^{k} \theta_{L}\right)_{\alpha_{1}}\left(\theta_{L} \gamma^{l m_{1} m_{2}} \theta_{L}\right)\left(\theta_{L} \gamma^{m_{3} m_{4}} \gamma^{p_{1} \cdots p_{3}} \gamma^{m_{5} m_{6}>} \theta_{L}\right) \\
= & \left(\mathcal{T}_{L}^{-1}\right)_{\left[\rho_{1} \cdots \rho_{5}\right]}^{\left(\left(\alpha_{1} \cdots \alpha_{3}\right)\right)}\left(\gamma_{p_{1} \cdots p_{3}}{ }^{<m_{7} m_{8}}\right)_{\alpha_{2} \alpha_{3}}\left(\gamma^{k}\right)_{\alpha_{1}}{ }^{\left[\rho_{1}\right.}\left(\gamma^{l m_{1} m_{2}}\right)^{\rho_{2} \rho_{3}}\left(\gamma^{m_{5} m_{6}} \gamma^{p_{1} \cdots p_{3}} \gamma^{m_{7} m_{8}>}\right)^{\left.\rho_{4} \rho_{5}\right]} .
\end{aligned}
$$

The tensors $\mathcal{T}_{L, R}^{-1}$ are given by the tree-level normalization (2.15):

$$
\begin{aligned}
& \left(\mathcal{T}_{L}^{-1}\right)_{\left[\rho_{1} \cdots \rho_{5}\right]}^{\left(\left(\alpha_{1} \cdots \alpha_{3}\right)\right)}=\left\langle\lambda^{\alpha_{1}} \lambda^{\alpha_{2}} \lambda^{\alpha_{3}} \theta_{\rho_{1}} \cdots \theta_{\rho_{5}}\right\rangle_{g=0} \\
& =\prod_{\beta_{1} \cdots \beta_{3}}^{\alpha_{1} \cdots \alpha_{3}}\left(\gamma^{n_{1}}\right)^{\left(\beta_{1}\right.}{ }_{\left[\rho_{1}\right.}\left(\gamma^{n_{2}}\right)^{\beta_{2}{ }_{\rho_{2}}}\left(\gamma^{n_{3}}\right)^{\left.\beta_{3}\right)}{ }_{\rho_{3}}\left(\gamma^{n_{1} \cdots n_{3}}\right)_{\left.\rho_{4} \rho_{5}\right]},
\end{aligned}
$$

where

$$
\Pi_{\beta_{1} \cdots \beta_{3}}^{\left(\left(\alpha_{1} \cdots \alpha_{3}\right)\right)}=\delta_{\beta_{1}}^{\left(\alpha_{1}\right.} \cdots \delta_{\beta_{3}}^{\left.\alpha_{3}\right)}-\frac{1}{6}\left(\gamma^{m}\right)^{\left(\alpha_{1} \alpha_{2}\right.} \delta_{\left(\beta_{1}\right.}^{\left.\alpha_{3}\right)}\left(\gamma_{m}\right)_{\left.\beta_{2} \beta_{3}\right)}
$$

projects on $\gamma_{m}$-traceless symmetric tensors, i.e. $\left(\gamma_{n}\right)_{\alpha_{1} \alpha_{2}} \Pi_{\beta_{1} \cdots \beta_{3}}^{\alpha_{1} \cdots \alpha_{3}}=0$.

Note that the tensor $t_{L}$, defined as the one-loop saturation of zero modes in (6.11), can also be written as a tree-level correlator in the following way:

$$
t_{L}^{k l \mid m_{1} \cdots m_{8}}=\left\langle\left(\lambda \gamma^{k} \theta\right)\left(\theta \gamma^{l<m_{1} m_{2}} \theta\right)\left(\lambda \gamma_{p_{1} \cdots p_{3}}{ }^{m_{3} m_{4}} \lambda\right)\left(\theta \gamma^{m_{5} m_{6}} \gamma^{p_{1} \cdots p_{3}} \gamma^{m_{7} m_{8}>} \theta\right)\right\rangle_{g=0} .
$$

We only need the contractions of the $k l$ indices of this tensor with the metric and with the $B$-field. To bring the expression for $t_{L}$ in a manageable form, we utilize the Fierz identity

$$
-\frac{1}{3 ! 16}\left(\lambda \gamma_{p_{1} \cdots p_{3}}{ }^{m_{1} m_{2}} \lambda\right)\left(\theta \gamma_{m_{3} m_{4}} \gamma^{p_{1} \cdots p_{3}} \gamma_{m_{5} m_{6}} \theta\right)=\left(\lambda \gamma^{\left[m_{1}\right.} \gamma_{m_{3} m_{4}} \theta\right)\left(\lambda \gamma^{\left.m_{2}\right]} \gamma_{m_{5} m_{6}} \theta\right) .
$$

Then we use the following identities:

$$
\begin{aligned}
\left\langle\left(\lambda \gamma^{m_{1} \cdots m_{3}} \theta\right)\left(\lambda \gamma^{m_{4} \cdots m_{6}} \theta\right)\left(\lambda \gamma^{m_{7}} \theta\right)\left(\theta \gamma_{m_{8} \cdots m_{10}} \theta\right)\right\rangle_{g=0} & = \pm \frac{1}{3 !} \epsilon_{10}^{m_{1} \cdots m_{10}}, \\
\left\langle\left(\lambda \gamma^{m_{1}} \theta\right) \cdots\left(\lambda \gamma^{m_{3}} \theta\right)\left(\theta \gamma_{n_{1} \cdots n_{3}} \theta\right)\right\rangle_{g=0} & =\frac{1}{120} \delta_{n_{1} \cdots n_{3}}^{m_{1} \cdots m_{3}}, \\
\left\langle\left(\lambda \gamma^{m} \theta\right)\left(\lambda \gamma^{n} \theta\right)\left(\lambda \gamma^{p_{1} \cdots p_{3}} \theta\right)\left(\theta \gamma_{q_{1} \cdots q_{3}} \theta\right)\right\rangle_{g=0} & =\frac{1}{30}\left(\delta_{n q_{1} \cdots q_{3}}^{m p_{1} \cdots p_{3}}-\delta_{m q_{1} \cdots q_{3}}^{n p_{1} \cdots p_{3}}-\frac{1}{4} \delta_{n}^{m} \delta_{q_{1} \cdots q_{3}}^{p_{1} \cdots p_{3}}\right) .
\end{aligned}
$$

The sign in the first equation is determined by the chirality of the ten-dimensional complex Weyl fermions $\theta$. With the help of (6.15)-(6.16) and (A.9), it is not difficult to check that

$$
\begin{aligned}
g_{k l} t_{L, R}^{k l \mid m_{1} \cdots m_{8}} & =t_{8}^{m_{1} \cdots m_{8}} \\
B_{k l} t_{L, R}^{k l \mid m_{1} \cdots m_{8}} & =B_{k l}\left(t_{10}^{k l m_{1} \cdots m_{8}} \pm \epsilon_{10}^{k l m_{1} \cdots m_{8}}\right) .
\end{aligned}
$$


The tensor $t_{10}$ is only composed of $\eta_{M N}$ 's and contracting it with a set of generic two-forms $F_{m n}^{i}, i=1, \ldots, 4$, gives

$$
t_{10}^{k l m_{1} \cdots m_{8}} B_{k l} F_{m_{1} m_{2}}^{1} \cdots F_{m_{7} m_{8}}^{4}=a_{1} \operatorname{tr}\left(B F^{1}\right) \operatorname{tr}\left(F^{2} F^{3} F^{4}\right)+a_{2} \operatorname{tr}\left(B F^{1} \cdots F^{4}\right)+\text { perms },
$$

symmetrized in the exchange of the $F^{i}$. However, the antisymmetry of $F^{i}$ and $B$ makes this expression to vanish. The sign in front of the $\epsilon_{10}$-term is related to the chirality of the $\theta$ variables. Since for type IIA/IIB $\theta_{L}$ and $\theta_{R}$ have the opposite/same chiralities, by antisymmetry of the $B$-field the amplitude (6.6) vanishes for type IIB and gives the $B \wedge t_{8} t_{8} R^{4}$ term for type IIA.

\section{$6.2 C_{3} \wedge X_{8}$ in eleven dimensions}

The zero mode counting of Section 5 shows that the first non zero amplitudes are with four external states. In particular, the one-loop four-graviton scattering, that produces the $R^{4}$ term, is non vanishing. ${ }^{16}$ In this subsection we will be interested in the supersymmetric partner of the latter, namely the five-point amplitude with four gravitons and a $C_{3}$-field.

The relevant vertex operators are restrictions of the vertex operators given in Subsection 3.1 to constant curvature $R_{M N P Q}$. The graviton vertex operators are given by

$$
\begin{aligned}
V_{R} & =\int d \tau R_{M N P Q} \hat{\mathcal{L}}^{M N} \hat{\mathcal{L}}^{P Q} e^{i k \cdot X} \\
U_{R}^{(1)} & =R_{M N P Q} \hat{\mathcal{L}}^{M N}\left(\lambda \Gamma^{R} \theta\right)\left(\theta \Gamma_{R}{ }^{P Q} \theta\right) e^{i k \cdot X},
\end{aligned}
$$

where $\hat{\mathcal{L}}^{M N}$ stands again for the zero mode restriction of the Lorentz generators

$$
\hat{\mathcal{L}}^{M N}=N^{M N}+d \Gamma^{M N} \theta+\frac{i}{2} P_{L}\left(\theta \Gamma^{L M N} \theta\right)+P^{[M} x^{N]} .
$$

Unfortunatly the vertex operator for the $C_{3}$-field is not so simple because the superparticle is not charged under it. Looking at the vertex operators of section 3.1, and in particular at the expressions for the super-vielbein components in (3.6), it appears that the field strength $G_{4}$ comes always in combination with the connection $\omega_{M}{ }^{r s}$ as

$$
V_{G_{4}+\omega}=\int d \tau\left[\omega_{M}^{r s}\left(d \Gamma_{r s} \theta\right)+\left(d \mathcal{T}_{M} \cdot G_{4} \theta\right)\right] P^{M} e^{i k \cdot X} .
$$

This means that the 5 points amplitude will read

$$
A_{5} \sim\left(G_{4}+\omega\right)^{2} R^{3} \sim C_{3} R^{4}+R^{4}+\left(G_{4}\right)^{2} R^{3},
$$

\footnotetext{
${ }^{16}$ This and related amplitudes will be discussed elsewhere [36].
} 
which shows that supersymmetry relates automatically the $R^{4}$ term with the ChernSimons $C_{3} \wedge t_{8} R^{4}$ one. $^{17}$

As an illustration we consider the amplitude composed of three integrated vertex operators for the graviton, one for the spin-connection, one for $G_{4}$ and one unintegrated vertex operator for the graviton. It is given by

$$
\begin{aligned}
\mathcal{A}= & R_{M_{1} M_{2} N_{1} N_{2}} \cdots R_{M_{5} M_{6} N_{5} N_{6}} \omega_{M_{7}}^{N_{7} N_{8}} G_{R_{1} \cdots R_{4}} \\
& \int[\mathcal{D} \lambda][\mathcal{D} w] \mathrm{d}^{32} \theta \mathrm{d}^{32} d \int \frac{d \tau}{\tau} b \prod_{i=1}^{4} d \tau_{i} Z_{J} \prod_{I=2}^{22} Z_{B} \prod_{I=1}^{32} Y_{C} \\
& \mathcal{M}^{M_{1} M_{2}} \cdots \mathcal{M}^{M_{5} M_{6}} \mathcal{M}^{N_{1} N_{2}} \mathcal{M}^{N_{3} N_{4}} \\
& \left(\lambda \Gamma^{R} \theta\right)\left(\theta \Gamma^{R N_{5} N_{6}} \theta\right) P^{M_{7}} N^{N_{7} N_{8}}\left(d \mathcal{T}_{S}^{R_{1} \cdots R_{4}} \theta\right) P^{S} \prod_{1 \leq i<j \leq 5} e^{i k^{(i)} \cdot k^{(j)} G_{i j}},
\end{aligned}
$$

where, as for the superstring, only the part

$$
\mathcal{M}^{M N}=N^{M N}+\left(d \Gamma^{M N} \theta\right)
$$

of the Lorentz generator $\hat{\mathcal{L}}^{M N}$ contributes from the integrated vertex operators. The $b$-ghost has to soak up the zero modes of one Lorentz generator [12], which we choose to take from the vertex operator for the spin connection. Using that for the on-shell superparticule $P^{M}=\dot{X}^{M}$ and also the world-line expression for the one-loop propagator [13

$$
\left\langle X^{M}\left(\tau_{i}\right) X^{N}\left(\tau_{j}\right)\right\rangle_{g=1}=\eta^{M N} G_{i j}=\frac{\eta^{M N}}{2}\left(\left|\tau_{i}-\tau_{j}\right|-\left(\tau_{i}-\tau_{j}\right)^{2}+\text { const. }\right)
$$

and integrating over the position of the vertex operators, we obtain the following result for the amplitude at leading order in the momenta

$$
\mathcal{A}=\int \frac{d \tau}{\tau^{3 / 2}} t_{11}^{S M_{1} \cdots M_{6} R_{1} \cdots R_{4} N_{1} \cdots N_{8}} R_{M_{1} M_{2} N_{1} N_{2}} \cdots R_{M_{5} M_{6} N_{5} N_{6}} \omega_{S}{ }^{N_{7} N_{8}} G_{R_{1} \cdots R_{4}}
$$

with

$$
\begin{aligned}
& t_{11}^{S M_{1} \cdots M_{6} R_{1} \cdots R_{4} N_{1} \cdots N_{8}}=\int[\mathcal{D} \lambda][\mathcal{D} w] \mathrm{d}^{32} \theta \mathrm{d}^{32} d \int b Z_{J} \prod_{I=2}^{22} Z_{B} \prod_{I=1}^{32} Y_{C} \\
& \times\left[N^{N_{7} N_{8}} \mathcal{M}^{M_{1} M_{2}} \cdots \mathcal{M}^{M_{5} M_{6}} \mathcal{M}^{N_{1} N_{2}} \mathcal{M}^{N_{3} N_{4}}\left(\lambda \Gamma^{R} \theta\right)\left(\theta \Gamma^{R N_{5} N_{6}} \theta\right)\left(d \mathcal{T}_{M_{7}}{ }^{R_{1} \cdots R_{4}} \theta\right)+\text { sym. }\right]
\end{aligned}
$$

where symmetrization between the $\left(M_{2 i-1}, M_{2 i}\right)$ and $\left(N_{2 i-1}, N_{2 i}\right)$ pairs is understood. The $b$-ghost insertion requires one $N^{M N}$ zero mode from the Lorentz generator $\mathcal{M}^{M N}$ and

\footnotetext{
${ }^{17}$ Note that $R^{4}$ contains two contributions: one is the non-linear completion of the four-point $t_{8} t_{8} R^{4}$ term and the other gives $\epsilon_{11} \epsilon_{11} R^{4}$.
} 
we have to get $5 d$ 's and $9 \theta$ 's to saturate the fermionic zero modes. Similarly to the superstring, with the help of (4.6) we can rewrite this tensor as a tree-level correlator

$$
\begin{aligned}
& t_{11}^{S M_{1} \cdots M_{6} R_{1} \cdots R_{4} N_{1} \cdots N_{8}}=\left\langle\left(\lambda \Gamma^{R} \theta\right)\left(\theta \Gamma^{R N_{1} N_{2}} \theta\right)\right. \\
\times & \left(\lambda \Gamma^{N_{3} N_{4}} \Gamma_{P_{1} \cdots P_{5}} \Gamma^{N_{5} N_{6}} \lambda\right)\left(\lambda \Gamma_{P_{6} P_{7}} \lambda\right)\left(\lambda \Gamma_{P_{8} P_{9}} \lambda\right) \\
\times & \left.\left(\theta \mathcal{T}_{M_{7}}^{R_{1} \cdots R_{4}} \Gamma^{P_{1} \cdots P_{3}} \Gamma^{N_{7} N_{8}} \theta\right)\left(\theta \Gamma^{M_{1} M_{2}} \Gamma^{P_{4} \cdots P_{6}} \Gamma^{M_{3} M_{4}} \theta\right)\left(\theta \Gamma^{M_{5} M_{6}} \Gamma^{P_{7} \cdots P_{9}} \Gamma^{M_{7} M_{8}} \theta\right)\right\rangle_{g=0},
\end{aligned}
$$

symmetrized over all exchanges of the pairs $\left(M_{2 i-1}, M_{2 i}\right)$ and $\left(N_{2 i-1}, N_{2 i}\right)$ for $i=1, \ldots, 4$. By Fierzing and using the tree-level normalization (2.15), one can see that the last expression has an expansion of the following general form:

$$
\sum_{\substack{n_{1}+n_{2}+\cdots=8 \\ n_{i} \geq 0}} c_{\left\{n_{i}\right\}} \operatorname{tr}\left(\left(\Gamma_{[2]}\right)^{n_{1}}\right) \cdots \operatorname{tr}\left(\left(\Gamma_{[2]}\right)^{n_{7}}\right) \operatorname{tr}\left(\left(\Gamma_{[2]}\right)^{n_{8}} \mathcal{T}_{S}^{R_{1} \cdots R_{4}}\right)
$$

where $\Gamma_{[2]}$ represents any of $\Gamma^{\left[M_{2 i-1} M_{2 i}\right]}$ or $\Gamma^{\left[N_{2 i-1} N_{2 i}\right]}$. The last multiplier in this expression is nonzero only for $n_{8}=4$, picking the $\eta_{M_{7}}^{\left[R_{1}\right.} \Gamma^{\left.R_{2} \cdots R_{4}\right]}$ from $\mathcal{T}_{M_{7}}{ }^{R_{1} \cdots R_{4}}$, to give an eleven

dimensional $\epsilon$-tensor $\epsilon_{11}^{R_{2} \cdots R_{4} \cdots}$. Integrating by parts the derivative on the four-form and relabelling the pairs of $M$ and $N$ indices, we obtain for the amplitude

$$
\mathcal{A}=\epsilon_{11}^{M_{1} \cdots M_{11}} C_{M_{9} \cdots M_{11}} R_{M_{1} M_{2} N_{1} N_{2}} \cdots R_{M_{7} M_{8} N_{7} N_{8}}\left(\operatorname{tr}\left(\left(\Gamma^{N N}\right)^{4}\right)-\frac{1}{16}\left(\operatorname{tr}\left(\Gamma^{N N}\right)^{2}\right)^{2}\right) .
$$

Finally using $(\mathrm{A} .10)$ with $\Delta=32$, we find that this amplitude gives $C_{3} \wedge t_{8} R^{4}$.

Obtaining the correct structure is very satisfying, but we should note that we haven't addressed here the issue of the UV regularization of the $11 d$ answer. In the light-cone computation of [7] it was resolved by considering M-theory on $R^{(8,1)} \times T^{2}$ and requiring that the reductions to IIA and IIB give T-dual answers. In the present pure spinor formulation such a program is a nontrivial task and we intend to come back to it in the future.

\subsection{Open string amplitudes in ten dimensions}

In the previous subsections we verified the correctness of the definition of the one-loop measure by recovering the known $B \wedge R^{4}$ and $C_{3} \wedge R^{4}$ terms in closed srting theory and eleven-dimensional supergravity respectively. In the current subsection we provide yet another check on the measure by comparing with known results for a simpler case, namely one loop in 10d open string theory. More precisely, we compute the supersymmetric $\mathcal{F}^{4}$ contribution to the effective action. By that we mean all three terms: four gluons; two gluons and two gluinos; four gluinos. 
Again we restrict ourselves to the massless states. The general expression for the covariant supervertex operator of 10d Super Yang-Mills is ${ }^{18}$

$$
\mathcal{V}^{(0)}=\dot{\theta}^{\alpha} A_{\alpha}(x, \theta)+\Pi^{m} A_{m}(x, \theta)+d_{\alpha} W^{\alpha}(x, \theta)+\left(w \gamma^{m n} \lambda\right) F_{m n}(x, \theta) .
$$

Using the field equations and Bianchi identities, one can derive the relations [37, 31]:

$$
\begin{aligned}
& D_{(\alpha} A_{\beta)}=\gamma_{\alpha \beta}^{m} A_{m} \\
& D_{\alpha} A_{m}-\partial_{m} A_{\alpha}=\gamma_{m \alpha \beta} W^{\beta} \\
& D_{\alpha} W^{\beta}=\frac{1}{4} \gamma_{\alpha}^{m n} F_{m n} \\
& D_{\alpha} F_{m n}=\left(\gamma_{[m} \partial_{n]} W\right)_{\alpha} .
\end{aligned}
$$

Together with the gauge choice

$$
\theta^{\alpha} A_{\alpha}=0,
$$

equations (6.29) allow one to obtain the full expansion in powers of $\theta$ of the superfields $A_{\alpha}, A_{m}, W^{\alpha}$ and $F_{m n}$ following the iterative procedure of [31, 43]. The only input is the identification of the lowest components as

$$
\left.A_{m}\right|_{\theta=0}=a_{m}(x),\left.\quad W^{\alpha}\right|_{\theta=0}=u^{\alpha}(x),\left.\quad F_{m n}\right|_{\theta=0}=f_{m n},
$$

where $a_{m}$ is the gauge field and $u^{\alpha}$ is the gluino. Using that for a constant field strength $a_{m}=f_{m n} x^{n}$, one finds for the first few terms of these expansions:

$$
\begin{aligned}
A_{\alpha} & =a_{m}\left(\gamma^{m} \theta\right)_{\beta}+\left(\gamma^{m} \theta\right)\left(u \gamma_{m} \theta\right)+\ldots \\
A_{m} & =a_{m}+u \gamma^{m} \theta+f_{p q} \theta \gamma^{m p q} \theta+\ldots \\
W^{\alpha} & =u^{\alpha}+\frac{1}{4} f_{m n}\left(\gamma^{m n} \theta\right)^{\alpha}+\cdots \\
F_{m n} & =f_{m n}-\theta \gamma_{[m} \partial_{n]} u+\cdots
\end{aligned}
$$

Substituting the last equations in (6.28), we obtain for the supervertex operator (on shell i.e. using also $\dot{\theta}=0$ and $\left.\dot{x}^{m}=P^{m}\right)$ :

$$
\mathcal{V}^{(0)}=u^{\alpha} q_{\alpha}+f_{m n}\left(\mathcal{M}^{m n}+P^{[m} x^{n]}\right)-\left(\theta \gamma_{[m} \partial_{n]} u\right)\left(\frac{1}{2} \mathcal{M}^{m n}+\frac{1}{3} P_{p} \theta \gamma^{p m n} \theta\right)
$$

where $\mathcal{M}^{m n}$ is defined as in (6.8) and $q_{\alpha}$ is the supersymmetry generator.

Now we will consider the four-point amplitude at one loop. Our goal will be to check if the entire effective SYM action to order $\alpha^{\prime 2}$ [17] is reproduced from the computational

\footnotetext{
${ }^{18}$ For simplicity we take the lowest component of the superfield $F_{m n}$, which is the gauge field strength, to be constant.
} 
rules of the pure spinor formalism. There are three cases for the external particles: four gluons; two gluons and two gluinos; four gluinos. We will handle all of them in turn.

For the case of four external gauge fields we take the following vertex operators: one $U_{f}^{(1)}=f_{p q} \lambda \gamma_{r} \theta \theta \gamma^{p q r} \theta$ and three $\mathcal{V}_{f}^{(0)}=f_{p q}\left(\mathcal{M}^{p q}+P^{[p} x^{q]}\right)$. Using the results of the previous sections, the one-loop prescription gives at leading order in the $\alpha^{\prime}$ expansion

$$
\begin{aligned}
& \left\langle Z_{J} \prod_{1}^{10} Z_{B} \prod_{1}^{11} Y_{C}(\lambda, \theta) \times\left[G^{\alpha}+H^{\alpha \beta} D_{\beta}+K^{\alpha \beta \gamma} D_{\beta} D_{\gamma}+L^{\alpha \beta \gamma \delta} D_{\beta} D_{\gamma} D_{\delta}\right] \frac{\partial}{\partial \lambda^{\alpha}}\right. \\
& \left.U_{f}^{(1)}(\lambda, \theta)\left(\int d \tau \mathcal{V}_{f}^{(0)}\right)^{3}\right\rangle=t_{8} f^{4}+\mathcal{O}\left(\alpha^{\prime 2}\right),
\end{aligned}
$$

which coincides exactly with the $f^{4}$ term in (3.7) of 17].

To describe the interaction of two gauge fields and two gluinos, in addition to a $U_{f}^{(1)}$ and a $\mathcal{V}_{f}^{(0)}$ we also need two gluino vertex operators $\mathcal{V}_{u}^{(0)}=u^{\alpha} q_{\alpha}-\left(\theta \gamma_{[m} \partial_{n]} u\right)\left(\frac{1}{2} \mathcal{M}^{m n}+\right.$ $\left.\frac{1}{3} P_{p} \theta \gamma^{p m n} \theta\right)$. By counting the zero modes of the various fields, it is easy to convince oneself that the only nonzero contribution comes from

$$
f_{p q} u^{\alpha}\left(\gamma_{m} \partial_{n} u\right)_{\beta}\left\langle\left\langle U_{f}^{(1)} \mathcal{M}^{p q} \theta^{\beta} \frac{1}{2} \mathcal{M}^{m n} q_{\alpha}\right\rangle\right\rangle_{g=1}
$$

where actually only the $p \theta$ term of $\mathcal{M}$ contributes. In (6.35) we have abbreviated by $\langle\langle\ldots\rangle\rangle$ the full one-loop measure, including the picture changing operators and the $b$-ghost. This last expression gives the effective interaction

$$
\mathcal{L}_{f^{2} u^{2}}=-2\left[f_{i j} f_{p q} u^{\alpha}\left(\gamma_{m} \partial_{n} u\right)_{\beta}\right] \delta^{m[i \mid} \delta^{n[p} \delta^{q] \mid j]} \delta_{\alpha}{ }^{\beta},
$$

which matches precisely the corresponding term in (3.7) of 17.

Finally, we turn to the four-gluino one-loop amplitude. Now we need three $\mathcal{V}_{u}^{(0)}$ 's and one unintegrated vertex operator for $u^{\alpha}: U_{u}^{(1)}=\lambda \gamma^{m} \theta u \gamma_{m} \theta$. Following the same steps as before we find for the amplitude

$$
\begin{aligned}
\mathcal{L}_{u^{4}} & =u^{\beta} \partial_{n} u^{\gamma} \partial_{q} u^{\delta}\left\langle\left\langle\left(\gamma_{p} \theta\right)_{\delta} \frac{1}{2} \mathcal{M}^{p q}\left(\gamma_{m} \theta\right)_{\gamma} \frac{1}{2} \mathcal{M}^{m n} U_{u}^{(1)} q_{\beta}\right\rangle\right\rangle_{g=1} \\
& =u^{\alpha} u^{\beta} \partial_{n} u^{\gamma} \partial_{q} u^{\delta}\left(\frac{1}{180} \gamma_{\alpha \beta}^{a b c} \delta^{n q} \gamma_{\gamma \delta, a b c}+\frac{3}{10} \gamma_{\alpha \beta}^{a b c} \delta_{[a}^{n} \gamma_{\gamma \delta, b} \delta_{c]}^{q}\right)
\end{aligned}
$$

which again agrees with the corresponding term in (3.7) of 17.

\section{Equivalence between spinorial and BRST cohomology}

\subsection{Deformations and cohomology}

So far we have been reconstructing the effective action from scattering amplitudes. However, there is another way of achieving the same goal. Namely, one can study the possible 
deformations of the equations of motion, consistent with the symmetries of the theory, and then integrate them to terms in the effective action. This is essentially the spinorial cohomology approach of [16] - [19], pioneered back in [44].

For instance, in the case of SYM theory the presence of radiative corrections is equivalent to deforming the superspace constraints as [37, 39, 40, 38, 41, 42, 45, 46, 17]:

$$
F_{\alpha \beta}=D_{(\alpha} A_{\beta)}-\gamma_{\alpha \beta}^{m} A_{m}=J_{(\alpha \beta)} .
$$

The right hand side contains the symmetric gamma-traceless spinorial 2-form $J_{(\alpha \beta)}(W, F)$ which is a function of a gauge invariant combination of the spinorial field strength $W^{\alpha}$ and the curvature $F_{m n}$. In order that $J_{(\alpha \beta)}$ is a consistent deformation it must be closed with respect to the spinorial differential $D_{\alpha}$ :

$$
D_{(\alpha} J_{\beta \gamma)}=\gamma_{(\alpha \beta}^{m} J_{\gamma) m}
$$

where $J_{\gamma m}$ takes into account that the left hand side is projected on the gamma-traceless part. An efficient way to rewrite the above equation is to use the pure spinor BRST language. So we replace (7.1) and (7.2) with

$$
\left\{Q, U^{(1)}\right\}=U^{(2)}, \quad\left\{Q, U^{(2)}\right\}=0,
$$

where $U^{(1)}=\lambda^{\alpha} A_{\alpha}$ and $U^{(2)}=\lambda^{\alpha} \lambda^{\beta} J_{\alpha \beta}\left[U^{(1)}\right]$. The functional dependence of $U^{(2)}$ reminds that the components of this superfield depend upon the gauge invariant combinations of the superfields in $U^{(1)}$. Notice that $J_{(\alpha \beta)}$ automatically contains only the 5 -form part; the vector part is absent because of the pure spinor constraints [45, 46, 17]. Note also that if $U^{(2)}$ can be expressed as a $Q$-exact variation of some superfield $\Omega\left[U^{(1)}\right]$, then it can be reabsorbed into a complicated non-linear redefinition of the superfield $A_{\alpha}$. So, then the nontrivial $U^{(2)}$ 's are given by the cohomology classes of $H^{(2)}(Q)$.

In the same way, we can consider the case of $11 \mathrm{~d}$ supergravity. The (linearized) equations of motion can be written in terms of a gamma-traceless spinorial superfield $A_{A B C}$ as $\Gamma_{M N P Q R}^{A B} D_{A} A_{B C D}=0$ and $\Gamma_{M N}^{A B} D_{A} A_{B C D}=0$. The superfield $A_{A B C}$ defines a vertex operator $U^{(3)}=\lambda^{A} \lambda^{B} \lambda^{C} A_{A B C}$ from which it is easy to see the invariance under $\delta A_{A B C}=\Gamma_{(A B}^{M} \Omega_{C) M}$. Other possible deformations of the linearized equations of motion can be parametrized by a vertex operator of the form $U^{(4)}=\lambda^{A} \lambda^{B} \lambda^{C} \lambda^{D} G_{A B C D}\left[U^{(3)}\right]$ where the superfield $G_{A B C D}$ is the symmetric gamma-traceless part of the 4-superform $G$. Again the functional dependence of $U^{(4)}$ is through gauge invariant combinations of the fields appearing in the vertex $U^{(3)}$. Then, one has

$$
\left\{Q, U^{(3)}\right\}=U^{(4)}, \quad\left\{Q, U^{(4)}\right\}=0 .
$$


This implies that $U^{(4)} \in H^{(4)}(Q)$. This group corresponds to the spinorial cohomology group $H_{\tau}^{(0,4)}$ studied in [16, 18, 19, 20]. In the Berkovits' pure spinor language the components of $U^{(4)}$ are made out of the antifields of the supergravity fields [10]. Here the elements of the cohomology $H^{(4)}(Q)$ are viewed as functions of $U^{(3)}$. The linearized equations of motion for the antifields, obtained from the condition $Q U^{(4)}=0$, are [10]:

$$
\partial^{M} g_{M N}^{*}-\frac{1}{2} \partial_{N}\left(\eta^{M P} g^{M P}\right)=0, \quad \partial^{M} C_{M N P}^{*}=0 .
$$

At the linearized level one can identify fields with their antifields: $g_{M N}^{*}=g_{M N}, C_{M N P}^{*}=$ $C_{M N P}$. This has the effect of fixing the gauge symmetries of the physical fields since the equations of motion of the antifields are the gauge fixing conditions of the fields. The above identification fixes the harmonic gauge for the graviton and the $\partial^{M} C_{M N P}=0$ gauge for the three-form. In these gauges the equations of motion become $\partial^{2} g_{M N}=\partial^{2} C_{M N P}=$ 0 . It is interesting to note that in this language the $\mathcal{O}\left(\ell_{P}^{3}\right)$ topological deformation of the equations of motion studied in [20] corresponds to a non-linear correction to the identification between fields and antifields, namely

$$
\begin{aligned}
C_{M N P}^{*} & =C_{M N P}+\beta\left(\ell_{P}\right)^{3}\left(\Omega_{L}\right)_{M N P} \quad \text { with } \quad d \Omega_{L}=\operatorname{tr}(R \wedge R), \\
G_{M N P Q}^{*} & =G_{M N P Q}+\beta\left(\ell_{P}\right)^{3} \operatorname{tr}(R \wedge R) .
\end{aligned}
$$

At this point we can see the limitations of the superparticle approach as a zero mode approximation to the supermembrane. Namely, since the vertex operator $U^{(4)}$ never enters in the definition of tree-level and loop superparticle amplitudes, no information about the value of the parameter $\beta$ can be extracted from the present formalism. It is necessary to do a membrane amplitude computation or use topological arguments as in [47] to fix $\beta$.

\subsection{Spinorial cohomology from an extended BRST operator}

The spinorial cohomology group $H_{F}^{(0,4)}$ of 19 is defined by the action of a fermionic derivative $d_{F}[\omega]=\left[d_{1} \omega\right]$ on the elements of $H_{\tau}^{(0,4)}$ associated with the $\tau_{0}$ part of the full differential $d=d_{0}+d_{1}+\tau_{0}+\tau_{1}$. Here we recover the same cohomology by constructing an extended BRST operator out of the full $d$. We view this procedure as more complete than the somewhat artificial truncation to $\tau_{0}$, although as we show below the two definitions are ultimately equivalent.

To achieve the above goal, we introduce a new type of anticommuting vector ghost $\xi^{M}$ with the help of which we can convert the differential $d$ into a BRST operator. In 
curved space the four parts of $d$ act as:

$$
\begin{aligned}
& \left(d_{0} \omega\right)_{M_{1} \ldots M_{p+1} A_{1} \ldots A_{q}}=\nabla_{\left[M_{1}\right.} \omega_{\left.M_{2} \ldots M_{p+1}\right] A_{1} \ldots A_{q}}+\frac{p}{2} T_{\left[M_{1} M_{2}\right.}{ }^{R} \omega_{\left.|R| M_{3} \ldots M_{p+1}\right] A_{1} \ldots A_{q}} \\
& +q(-1)^{p} T_{\left[M _ { 1 } \left(A_{1}\right.\right.}{ }^{C} \omega_{\left.\left.M_{2} \ldots M_{p+1}\right]|C| A_{2} \ldots A_{q}\right)} \\
& \left(d_{1} \omega\right)_{M_{1} \ldots M_{p} A_{1} \ldots A_{q+1}}=(-1)^{p} \nabla_{\left(A_{1}\right.} \omega_{\left.M_{1} \ldots M_{p} A_{2} \ldots A_{q+1}\right)}+\frac{q}{2} T_{\left(A_{1} A_{2}\right.}{ }^{C} \omega_{\left.\left.M_{1} \ldots M_{p}\right]|C| A_{3} \ldots A_{q+1}\right)} \\
& +p(-1)^{p} T_{\left(A _ { 1 } \left[M_{1}\right.\right.}{ }^{R} \omega_{\left.\left.|R| M M_{2} \ldots M_{p}\right] A_{2} \ldots A_{q+1}\right)} \\
& \left(\tau_{0} \omega\right)_{M_{1} \ldots M_{p-1} A_{1} \ldots A_{q+2}}=\frac{p}{2} T_{\left(A_{1} A_{2}\right.}{ }^{R} \omega_{\left.|R| M_{1} \ldots M_{p-1} A_{3} \ldots A_{q+2}\right)} \\
& \left(\tau_{1} \omega\right)_{M_{1} \ldots M_{p+2} A_{1} \ldots A_{q-1}}=\frac{q}{2} T_{\left[M_{1} M_{2}\right.}{ }^{B} \omega_{\left.\left.M_{3} \ldots A_{p+2}\right]|B| A_{1} \ldots B_{q-1}\right)},
\end{aligned}
$$

where $\nabla$ is the covariant derivative, $\omega$ is a supeform belonging to $\Omega^{(p, q)}$ and $T_{\Omega \Sigma}{ }^{\Theta}$ is the supertorsion. We consider flat space and therefore the only non-vanishing components of the latter are $T_{A B}{ }^{M}=-i \gamma_{A B}{ }^{M}$.

Contracting the different pieces of the differential operator $d$ with the ghost fields $\lambda^{A}$ and $\xi^{M}$ (and the conjugate momenta $w_{A}, \beta_{M}$ ) one finally gets the BRST operator

$$
\begin{aligned}
Q=\sum_{n=-1}^{2} Q_{n} & =\lambda^{A} \lambda^{B} T_{A B}^{R} \beta_{R}+\left(\lambda^{A} \lambda^{B} T_{A B}^{C} w_{C}+\lambda^{A} \xi^{N} T_{A N}^{R} \beta_{R}+\lambda^{A} \nabla_{A}\right)+ \\
& +\left(\xi^{M} \lambda^{A} T_{M A}^{B} w_{B}+\xi^{M} \xi^{N} T_{M N}^{R} \beta_{R}+\xi^{M} \nabla_{M}\right)+\xi^{M} \xi^{N} T_{M N}^{A} w_{A},
\end{aligned}
$$

where the index $n$ of the different pieces of $Q$ denotes the grading obtained by summing the gradings of the corresponding ghost fields. The latter have gradings zero for $\lambda^{A}, w_{A}$ and $(1,-1)$ for $\xi^{M}, \beta_{M}$. In the present context the grading coincides with the engineering dimension of the variables $P_{M}$ and $d_{A}$. Due to the properties of the covariant derivatives and by the defintion of the supertorsion $T$, one can check that $Q$ is nilpotent.

The forms of type $(p, q)$ are mapped into vertex operators $U^{(p, q)}$ with $p \lambda^{A}$-ghosts and $q \xi^{M}$-ghosts and the action of the BRST operator is

$$
Q: U^{(p, q)} \rightarrow U^{(p+2, q-1)} \oplus U^{(p+1, q)} \oplus U^{(p, q+1)} \oplus U^{(p-1, q+2)} .
$$

The total grading is given by $q$. Notice that the space has a double filtration: the grading number (which is given by the number $\xi^{M}$ ghosts) and the total ghost number (form number) which is given by the sum $p+q$. The BRST operator $Q$ raises by one unit the ghost number. It is easy to check that, due to the Poincaré lemma, all the cohomology 
groups $H^{(p, q)}(Q)$ are empty, except $H^{(0,0)}(Q)$ which contains only the constant functions. So any $Q$-closed form is also $Q$-exact.

On the other hand, the double filtration means that it is possible to introduce a new BRST operator which has a definite grading number, is nilpotent and has vanishing cohomology. For that purpose, we introduce a new pair of ghosts $(c, b)$ with ghost numbers $(1,-1)$ and grading numbers $(1,-1)$ and we define the new BRST operator as $Q^{\prime}=c$. This is clearly the simplest possible choice, but it has all the correct properties. The space of forms has to be enlarged to contain the ghost field $b$. In this way the vertex operators $U^{(p, q)}$ might have even negative grading. ${ }^{19}$ It is easy to see that the cohomology of $Q^{\prime}$ is empty.

Then, we can consider the sum of the two BRST operators $Q+Q^{\prime}$ and restrict the space of vertex operators $U^{(p, q)}$ to those whose grading number $n$ is greater or equal to the number of anticommuting ghosts that they contain, i.e. $n \geq q$. Then we have:

$$
H^{(p, q)}(Q, \text { pure spinors }) \simeq H^{(p+q)}\left(Q+Q^{\prime}, n \geq q\right) .
$$

The right hand side of this equation defines correctly the spinorial cohomology discussed in [16, 17, 18, 19, 20]. Indeed, although the cohomology of $Q$ is empty due to the constraints coming from $Q_{(n)}$ with $n \geq 1$, the introduction of $Q^{\prime}$ allows to recover the pure spinor cohomology. The technique used here has been applied to the superstring in [21] in order to prove the equivalence of the BRST cohomologies with and without pure spinors 50.

We conclude the present section with a remark about the construction of different pictures in the pure spinor approach using standard methods from supergeometry. The space of forms $\Omega^{(p, q)}$ is not sufficient to define a sensible integration on supermanifolds. One has to consider the space of superforms of the type $\Omega_{m}^{(p, q, r)}$

$$
\xi^{M_{1}} \ldots \xi^{M_{p}} \lambda^{A_{1}} \ldots \lambda^{A_{q+m}} \partial_{\lambda_{A_{1}}} \ldots \partial_{\lambda_{A_{m}}} \prod_{k=1}^{r} \delta\left(C_{B}^{k} \lambda^{B}\right)
$$

where $r$ denotes the power of delta functions in the generalized forms and it corresponds to the picture number. For a given supermanifold $\mathbf{R}^{(m \mid n)}$ the picture number can run from

\footnotetext{
${ }^{19}$ The situation is very similar to the NSR superstring, where the BRST charge $Q$ is filtered with respect to the picture number. In the Large Hilbert Space, which contains the zero mode $\xi_{0}$ of the fermion $\xi$ (obtained from the fermionization of the $\beta, \gamma$ superghosts), the BRST operator $Q$ has no cohomology. However, one can introduce a second BRST differential $Q^{\prime}=\eta_{0}$ (namely, the zero mode of the conjugate momentum of $\xi_{0}$ ) with picture number +1 and positive ghost number, which is nilpotent, anticommutes with $Q$ and has no cohomology 48,49 . In addition, the vertex operators $U^{(p, q)}$ are labeled by both the ghost number and the picture number similarly to our superforms, which are labeled by a ghost number and a grading number. And again similarly to our case, one can see, that by considering the sum $Q+Q^{\prime}$ and restricting the functional space to finite combinations of $U^{(p, q)}, U^{(p)}=\sum_{q=n_{-}}^{n_{+}} U^{(p, q)}$, the cohomology is equivalent to the cohomology of $Q$ in the Small Hilbert Space.
} 
$r=0$ to $r=n+1$. For the two extreme values $r=0$ and $r=n+1$ one has that $n \geq 0$ and $n \leq m+1$ respectively whereas for $0<r<n+1$ the grading number $n$ can assume any value. Notice that the derivatives acting on the delta functions introduce negative spinorial degrees of the form. The differential $d$ maps in the space of forms without changing the picture. To change the picture, the operators $Z_{B}$ and $Y_{C}$ should be used. Their construction from pure geometrical concepts will be discussed in a forthcoming publication [51].

\section{Recapitulation and discussion}

In this paper we developed the rules for calculation of one-loop amplitudes for the elevendimensional superparticle. Using them and the string theory counterpart of [12], we computed covariantly the following one-loop terms in the 10- and 11- dimensional effective actions: $B \wedge X_{8}$ in IIA/B; $\mathcal{F}^{4}$ in type I; $C \wedge X_{8}$ in M-theory. We also verified that the eleven-dimensional supergravity action of [15] is reproduced by the tree-level scattering amplitudes, as should be the case for consistency. Hence, using the vertex operators $U^{(3)}$ and $U^{(1)}$ that were defined in Section 3.1, we can summarize the Cremmer-Julia-Scherk action by the expression (4.18):

$$
S_{C J S}=\left\langle U^{(3)} Q U^{(3)}\right\rangle+\left\langle U^{(3)}\left[U^{(1)}, U^{(3)}\right]\right\rangle,
$$

where the bracket $\langle\ldots\rangle$ is understood with the tree-level normalization $\left\langle\lambda^{7} \theta^{9}\right\rangle=1$, recalled in Section 2 , and [.,.] means taking the canonical commutators for the on-shell fields in the vertex operators.

It is possible to extend the present construction to multiloop amplitudes, in which case there will be more zero modes for the Lorentz generators. Also, as can be seen from the structure of the vertex operators in the new $b$-picture in (5.25), the tensors $K^{A B C}$ and $L^{A B C D}$ will contribute. As these terms are coming with extra fermionic derivatives, the corresponding amplitude will contain higher-derivatives. It would be interesting to try to reproduce the $D^{4} R^{4}$ terms analyzed in [9] from a two-loop amplitude for the superparticle.

However, being an approximation to the super-membrane for configurations with constant transverse excitations, the superparticle formalism clearly cannot grasp all information about the $\ell_{P}$-corrections to M-theory. In particular, we saw that because the membrane vertex operator for the anti-fields, $U^{(4)}$, does not enter in the definition of the tree and loop amplitudes for the superparticle, it is not possible to deduce any information about the $\left(\ell_{P}\right)^{3}$-deformations of the four-form field strength addressed in [20, 47]. 
Extension of the present work to the full supermembrane is not obvious. First of all, the BRST operator for the M2-brane requires new secondary constraints [10]:

$$
\left(\lambda \Gamma^{M N} \lambda\right) \Pi_{J M}\left(g, C_{3}\right)=0, \quad \lambda_{A} \partial_{J} \lambda^{A}=0 \quad \text { with } \quad \Pi_{J}^{M}=E_{\Omega}{ }^{M} \partial_{J} Z^{\Omega} \text { and } \quad J=1,2,
$$

involving the transverse excitations of the membrane. One can view the first constraint as a restriction not on the pure spinor but instead on the background metric $g$ and threeform $C_{3}$ in which the membrane evolves. It is interesting to notice that the last equation is not only algebraic but involves the dynamics of the pure spinors.

Another expected difficulty stems from the complicated nonlinear dynamics of the membrane excitations. In particular, even BPS amplitudes are not expected to be easy to understand based on the work of [52] which showed that, although the counting of membrane instantons wrapping $T^{3}$ can be obtained by computing the partition function of an associated 3d Matrix model, the model does not localize on the semi-classical configurations of the long membrane wrapping the $T^{3}$ torus.

\section{Acknowledgments}

The authors would like to thank the organizers of the second Simons Workshop in Stony Brook, 2004, for providing a very nice environment, where this paper could be completed, and for financial support. We would like to thank Nathan Berkovits for many useful discussions and for reading the manuscript. We are also grateful to M. Roček, W. Siegel and P. van Nieuwenhuizen for positive feedback. P.A.G. thanks the institute IHES, Buressur-Yvette, for hospitality during the initial stages of this work and also L. Castellani and A. Lerda for useful conversations. P.V. thanks the LPTHE of Jussieu and the Physics Department of Neuchâtel University for hospitality.

The research of L.A. was supported in part by DOE grant DE-FG02-95ER40899. The research of P.A.G. was partially supported by NSF-grant PHY-0354776. P.V. was partially supported by the EU networks HPRN-CT-2000-00148, and HPRN-CT-2000-00131.

\section{A. Fierz identities and Gamma matrix manipulations in 11d}

In this appendix we write down several Fierz identities that we need in the main text. We refer to [53] for an exhaustive list of the Fierz identities in eleven dimensions.

As Berkovits' pure spinors satisfy only (4.3) but not $\lambda^{A} \Gamma_{A B}^{M N} \lambda^{B}=0$, the Fierz identity between two $\lambda$ 's takes the form:

$$
32 \lambda^{(A} \lambda^{B)}=\frac{1}{2 !}\left(\Gamma^{M N}\right)^{A B}\left(\lambda \Gamma_{M N} \lambda\right)+\frac{1}{5 !}\left(\Gamma^{M_{1} \cdots M_{5}}\right)^{A B}\left(\lambda \Gamma_{M_{1} \cdots M_{5}} \lambda\right) .
$$


The $d^{23} N$-measure (5.11) is expanded on products of bilinears of $\lambda^{A}$ made from $\Gamma$ matrices with two, five and six indices. The relevant Fierz identities are:

$$
\begin{aligned}
\left(\Gamma^{M}\right)_{(A B}\left(\Gamma_{M N}\right)_{C D)} & =0 \\
\left(\Gamma_{M}\right)_{(A B}\left(\Gamma^{M N_{1} \cdots N_{4}}\right)_{C D)} & =6\left(\Gamma^{\left[N_{1} N_{2}\right.}\right)_{(A B}\left(\Gamma^{\left.N_{3} N_{4}\right]}\right)_{C D)}, \\
\left(\Gamma_{M}\right)_{(A B}\left(\Gamma^{M N_{1} \cdots N_{5}}\right)_{C D)} & =-\frac{1}{2} \delta_{(A B}\left(\Gamma^{N_{1} \cdots N_{5}}\right)_{C D)}-\frac{5}{2}\left(\Gamma^{\left[N_{1}\right.}\right)_{(A B}\left(\Gamma^{\left.N_{2} \cdots N_{5}\right]}\right)_{C D)} \\
& +5\left(\Gamma^{\left[N_{1} N_{2}\right.}\right)_{(A B}\left(\Gamma^{\left.N_{3} \cdots N_{6}\right]}\right)_{C D)}, \\
\left(\Gamma^{M_{1} M_{2}}\right)_{(A B}\left(\Gamma^{N_{1} \cdots N_{6}}\right)_{C D)} & =-\frac{1}{4} \delta_{(A B}\left(\Gamma^{M_{1} M_{2} N_{1} \cdots N_{6}}\right)_{C D)} \\
& +\frac{5}{2}\left(\Gamma^{\left(\left[M_{1} M_{2} N_{1} N_{2}\right.\right.}\right)_{(A B}\left(\Gamma^{\left.N_{3} \cdots N_{6}\right]}\right)_{C D)} .
\end{aligned}
$$

(A.1) is known as the M2-brane identity and (A.2) - as the M5-brane identity. These Fierz identities immediatly imply that:

$$
\begin{aligned}
\left(\lambda \Gamma_{M}\right)_{A}\left(\lambda \Gamma^{M N} \lambda\right)= & \\
\left(\lambda \Gamma_{M}\right)_{A}\left(\lambda \Gamma^{M N_{1} \cdots N_{4}} \lambda\right)= & \left(\lambda \Gamma^{\left[N_{1} N_{2}\right.}\right)_{A}\left(\lambda \Gamma^{\left.N_{3} N_{4}\right]} \lambda\right) \\
\left(\lambda \Gamma^{M_{1} M_{2}} \lambda\right)\left(\lambda \Gamma^{N_{1} \cdots N_{6}} \lambda\right)= & 0 \\
\lambda_{A}\left(\lambda \Gamma^{M_{1} \cdots M_{6}} \lambda\right)= & -6\left(\lambda \Gamma^{\left[M_{1}\right.}\right)_{A}\left(\lambda \Gamma^{\left.M_{2} \cdots M_{6}\right]} \lambda\right) \\
& +15\left(\lambda \Gamma^{\left[M_{1} M_{2}\right.} \lambda\right)\left(\lambda \Gamma^{\left.M_{3} \cdots M_{6}\right]}\right)_{A} .
\end{aligned}
$$

From (A.7) it follows that the measure factor for $d N$ in (5.11) cannot contain any terms of the form $\left(\lambda \Gamma_{[2]} \lambda\right)\left(\lambda \Gamma_{[6]} \lambda\right)$, where as in the main text we denote by $\Gamma_{[n]}$ the antisymmetric product of $n$ Gamma-matrices.

It is also useful to recall the explicit form of the $t_{8}$-tensor:

$$
\begin{aligned}
t_{8}^{m_{1} m_{2} n_{1} n_{2} p_{1} p_{2} q_{1} q_{2}} & =-2\left(\delta^{m_{1} n_{2}} \delta^{m_{2} n_{1}} \delta^{p_{1} q_{2}} \delta^{p_{2} q_{1}}+\delta^{n_{1} p_{2}} \delta^{n_{2} p_{1}} \delta^{m_{1} q_{2}} \delta^{m_{2} q_{1}}+\delta^{m_{1} p_{2}} \delta^{m_{2} p_{1}} \delta^{n_{1} q_{2}} \delta^{n_{2} q_{1}}\right) \\
& +8\left(\delta^{m_{1} q_{2}} \delta^{m_{2} n_{1}} \delta^{n_{2} p_{1}} \delta^{p_{2} q_{1}}+\delta^{m_{1} q_{2}} \delta^{m_{2} p_{1}} \delta^{p_{2} n_{1}} \delta^{n_{2} q_{1}}+\delta^{m_{1} n_{2}} \delta^{m_{2} p_{1}} \delta^{p_{2} q_{1}} \delta^{q_{2} n_{1}}\right) \\
& + \text { anti-sym. in every index pair. }
\end{aligned}
$$

In $d=10$ and $d=11$ it can be represented in terms of gamma-matrices as follows:

$$
\begin{aligned}
2 \Delta t_{8}^{m_{1} m_{2} n_{1} n_{2} p_{1} p_{2} q_{1} q_{2}} & =\operatorname{tr}\left(\Gamma^{m_{1} m_{2}} \Gamma^{m_{3} m_{4}} \Gamma^{m_{5} m_{6}} \Gamma^{m_{7} m_{8}}\right)+\operatorname{tr}\left(\Gamma^{m_{5} m_{6}} \Gamma^{m_{1} m_{2}} \Gamma^{m_{3} m_{4}} \Gamma^{m_{7} m_{8}}\right) \\
& +\operatorname{tr}\left(\Gamma^{m_{3} m_{4}} \Gamma^{m_{5} m_{6}} \Gamma^{m_{1} m_{2}} \Gamma^{m_{7} m_{8}}\right)-\frac{2}{\Delta} \operatorname{tr}\left(\Gamma^{m_{1} m_{2}} \Gamma^{m_{3} m_{4}}\right) \operatorname{tr}\left(\Gamma^{m_{5} m_{6}} \Gamma^{m_{7} m_{8}}\right) \\
& -\frac{2}{\Delta} \operatorname{tr}\left(\Gamma^{m_{1} m_{2}} \Gamma^{m_{5} m_{6}}\right) \operatorname{tr}\left(\Gamma^{m_{3} m_{4}} \Gamma^{m_{7} m_{8}}\right)-\frac{2}{\Delta} \operatorname{tr}\left(\Gamma^{m_{1} m_{2}} \Gamma^{m_{7} m_{8}}\right) \operatorname{tr}\left(\Gamma^{m_{3} m_{4}} \Gamma^{m_{5} m_{6}}\right),
\end{aligned}
$$

where $\Delta$ is the dimension of the Clifford algebra. 


\section{B. $\mathrm{SO}(8)$ parametrization of the 11d Pure Spinors}

In this appendix we explain the $S O(8)$ decomposition of an eleven-dimensional pure spinor, which makes manifest the number of independent components it has.

In Section 2 we introduced a commuting spinor $\lambda^{\prime}=\left(\lambda_{1}^{\prime}, \lambda_{2}^{\prime}\right)$ satisfying the constraint $\lambda^{\prime} \Gamma^{+} \lambda^{\prime}=0$, i.e. $\lambda_{1}^{\prime} \gamma^{+} \lambda_{1}^{\prime}+\lambda_{2}^{\prime} \gamma^{+} \lambda_{2}^{\prime}=0$. This constraint can be solved in the following way: decompose $\lambda_{1}^{\prime}$ into $s_{a}$ and $s_{\dot{a}}$, belonging to the $S O(8)$ representations $8_{c}$ and $8_{s}$ respectively. Then the equation $\lambda_{1}^{\prime} \gamma^{+} \lambda_{1}^{\prime}+\lambda_{2}^{\prime} \gamma^{+} \lambda_{2}^{\prime}=0$ becomes $s_{a} s_{a}=v^{+}$, where $v^{+} \equiv \lambda_{2}^{\prime} \gamma^{+} \lambda_{2}^{\prime}$ (the $S O(8)$ indices are raised and lowered with the metrics $\delta_{a b}$ and $\delta_{\dot{a} \dot{b}}$ ). The equation is preserved by $S O(7)$ rotations: using the triality to view $s_{a}$ as a vector of $S O(8)$ and decomposing it into the coset representation $S^{7} \times S O(7)$, one can fix its $8^{\text {th }}$ component to be equal to $\sqrt{v^{+}-s_{i} s_{i}}$, where $s_{i}$ is the $S O(7)$-part of $v$. Then, using the similarity transformation of Section 2, one can find the BRST operator $\lambda_{1}^{\alpha} d_{1 \alpha}+\lambda_{2 \alpha} d_{2}^{\alpha}$, where the 11d pure spinor is given by

$$
\lambda=\left(\lambda_{1}, \lambda_{2}\right)=\left(\left(\sqrt{v^{+}-s_{i}^{2}}, s_{i}\right),(0,0), \lambda_{2}^{\prime}\right) .
$$

The first two entries are the $s_{a}$ of $\lambda_{1}^{\prime}$, and the third and the fourth ones, $(0,0)$, are the 1 +7 components of $s_{a}$ with respect to $S O(7)$ using again triality. Finally, $\lambda_{2}^{\prime}$ is completely free. The counting of degrees of freedom gives $7+16=23$ (complex), which is exaclty the number of independent components of a pure spinor in 11d.

Notice that the above computation is parallel to the $10 \mathrm{~d}$ one of Berkovits. Indeed, in ten dimensions the $S O(9,1)$ spinor $\lambda_{\alpha}$ is reduced to two $S O(8)$ spinors, $\tilde{s}_{a}$ and $\tilde{s}_{\dot{a}}$. Then one imposes the pure spinor constraint and finds that $\tilde{s}_{a} \tilde{s}_{a}=0$. So the spinor $\gamma^{+} \lambda=\tilde{s}_{a}$ is null, i.e. $\lambda \gamma^{+} \lambda=0$. Then one can decompose the rest under a $S U(4)$ subgroup of $S O(8)$, which preserves the null property of $\tilde{s}_{a}$. Therefore the resulting solution for a pure spinor is

$$
\lambda=\left\{\tilde{s}_{a}, s_{A}, 0\right\},
$$

where $s_{A}$ is a generic spinor of $S U(4)$. Hence there are $4+7=11$ independent components, coming respectively from $s_{A}$ and $\tilde{s}_{a}$. Comparing with the above $11 \mathrm{~d}$ case, we see that $s_{A}$ replaces $\lambda_{2}^{\prime}$ whereas $\tilde{s}_{a}$ replaces $\left(s_{a}, 0\right)$ with $s_{a}-$ a spinor of $S^{7}$.

\section{References}

[1] P. Townsend, The Eleven-Dimensional Supermembrane Revisited, Phys. Lett. B350 (1995) 184, hep-th/9501068; E. Witten, String Theory Dynamics in Various Dimensions, Nucl. Phys. B443 (1995) 85, hep-th/9503124. 
[2] E. Bergshoeff, E. Sezgin, P. Townsend, Supermembranes and Eleven-Dimensional Supergravity, Phys. Lett. B189 (1987) 75.

[3] B. de Wit, M. Luscher and H. Nicolai, The Supermembrane Is Unstable, Nucl. Phys. B 320 (1989) 135.

[4] A. Dasgupta, H. Nicolai and J. Plefka, An introduction to the quantum supermembrane, Grav. Cosmol. 8 (2002) 1 [Rev. Mex. Fis. 49S1 (2003) 1] [arXiv:hep-th/0201182].

[5] A. Dasgupta, H. Nicolai and J. Plefka, Vertex operators for the supermembrane, JHEP 0005 (2000) 007 [arXiv:hep-th/0003280].

[6] T. Banks, W. Fischler, S. Shenker and L. Susskind, M-Theory as a Matrix Model: a Conjecture, Phys. Rev. D55, (1997) 5112, hep-th/9610043.

[7] M. Green, M. Gutperle, P. Vanhove, One Loop in Eleven Dimensions, Phys. Lett. B409 (1997) 177, hep-th/9706175.

[8] M. Green, M. Gutperle, Kwon, Quantum Mechanics of the Eleven-dimensional Superparticle, JHEP 9908 (1999) 012, hep-th/9907155.

[9] M. Green, H. Kwon, P. Vanhove, Two Loops in Eleven Dimensions, Phys. Rev. D61 (2000) 104010 [arXiv:hep-th/9910055].

[10] N. Berkovits, Towards Covariant Quantization of the Supermembrane, JHEP 0209 (2002) 051 [arXiv:hep-th/0201151].

[11] N. Berkovits, Super-Poincare covariant quantization of the superstring, JHEP 0004 (2000) 018 [arXiv:hep-th/0001035].

[12] N. Berkovits, Multiloop Amplitudes and Vanishing Theorems using the Pure Spinor Formalism for the Superstring, [arxiv: hep-th/0406055].

[13] M. J. Strassler, Field theory without Feynman diagrams: One loop effective actions, Nucl. Phys. B 385 (1992) 145 [arXiv:hep-ph/9205205].

[14] Z. Bern and D. Kosower, A new approach to one loop calculations in gauge theories, Phys. Rev. D38 (1988) 1888.

[15] E. Cremmer, B. Julia and J. Scherk, Supergravity Theory In 11 Dimensions, Phys. Lett. B 76 (1978) 409.

[16] M. Cederwall, U. Gran, M. Nielsen and B. E. W. Nilsson, Manifestly supersymmetric M-theory, JHEP 0010 (2000) 041 [arXiv:hep-th/0007035].

[17] M. Cederwall, B. Nilsson, D. Tsimpis, $D=10$ super-Yang-Mills at $O\left(\alpha^{\prime 2}\right)$, JHEP 0107 (2001) 042, hep-th/0104236. 
[18] M. Cederwall, B. E. W. Nilsson and D. Tsimpis, Spinorial cohomology and maximally supersymmetric theories, JHEP 0202 (2002) 009 [arXiv:hep-th/0110069].

[19] P. S. Howe and D. Tsimpis, On higher-order corrections in M theory, JHEP 0309 (2003) 038 [arXiv:hep-th/0305129].

[20] D. Tsimpis, 11D supergravity at $\mathcal{O}\left(l^{3}\right)$, arXiv:hep-th/0407271.

[21] P. A. Grassi, G. Policastro, M. Porrati and P. Van Nieuwenhuizen, Covariant quantization of superstrings without pure spinor constraints, JHEP 0210, 054 (2002) [arXiv:hep-th/0112162]; P. A. Grassi, G. Policastro and P. van Nieuwenhuizen, The massless spectrum of covariant superstrings, JHEP 0211, 001 (2002) [arXiv:hep-th/0202123].

[22] E. Cremmer and S. Ferrara, Eleven-Dimensional Supergravity in Superspace, Phys. Lett. B 91 (1980) 61.

[23] L. Brink and P. Howe, Eleven-Dimensional Supergravity on the Mass-Shell in Superspace Phys. Lett. B 91 (1980) 384.

[24] H. Nishino and S. Rajpoot, A note on embedding of M-theory corrections into eleven-dimensional superspace, Phys. Rev. D 64, 124016 (2001) [arXiv:hep-th/0103224].

[25] L. Brink and J. H. Schwarz, Quantum Superspace, Phys. Lett. B 100 (1981) 310.

[26] N. Berkovits, Covariant quantization of the superparticle using pure spinors, JHEP 0109 (2001) 016 [arXiv:hep-th/0105050].

[27] L. Anguelova and P. A. Grassi, Super D-branes from BRST symmetry, JHEP 0311 (2003) 010 [arXiv:hep-th/0307260].

[28] N. Berkovits, ICTP Lectures on Covariant Quantization of the Superstring, hep-th/0209059.

[29] B. de Wit, K. Peeters and J.C. Plefka, Superspace Geometry for supermembrane Background, Nucl. Phys. B 532 (1998) 99 [arXiv:hep-th/9803209].

[30] D. Tsimpis, Curved 11D Supergeometry, hep-th/0407244.

[31] J. Harnad and S. Shnider, Constraints and Field Equations for Ten-Dimensional Super-Yang-Mills Theory, Commun. Math. Phys. 106 (1986) 183.

[32] P. A. Grassi and L. Tamassia, Vertex operators for closed superstrings, arXiv:hep-th/0405072.

[33] M. Grisaru and M. Knutt, Norcor vs the abominable gauge completion, Phys. Lett. B500 (2001) 188, hep-th/0011173. 
[34] C. Vafa and E. Witten, A one loop test of string duality, Nucl. Phys. B447 (1995) 261 [arXiv:hep-th/9505053].

[35] M. Duff, J. Liu and R. Minasian, Eleven Dimensional Origin of String/String Duality: A One Loop Test, Nucl. Phys. B452 (1995) 261, hep-th/9506126.

[36] L. Anguelova, P.A. Grassi and P. Vanhove, Work in progress.

[37] E. Witten, Twistor - Like Transform In Ten-Dimensions, Nucl. Phys. B 266 (1986) 245.

[38] B. E. W. Nilsson, Off-Shell $D=10, N=1$ Poincare Supergravity And The Embeddibility Of Higher Derivative Field Theories In Superspace, Phys. Lett. B 175 (1986) 319.

[39] B. E. W. Nilsson, Pure Spinors As Auxiliary Fields In The Ten-Dimensional Supersymmetric Yang-Mills Theory, Class. Quant. Grav. 3, L41 (1986).

[40] J. J. Atick, A. Dhar and B. Ratra, Superstring Propagation In Curved Superspace In The Presence Of Background Superyang-Mills Fields, Phys. Lett. B 169 (1986) 54.

[41] S. J. . Gates and H. Nishino, Manifestly Supersymmetric O (Alpha-Prime) Superstring Corrections In New $D=10, N=1$ Supergravity Yang-Mills Theory, Phys. Lett. B 173 (1986) 52.

[42] S. J. J. Gates and S. Vashakidze, On $D=10, N=1$ Supersymmetry, Superspace Geometry And Superstring Effects, Nucl. Phys. B 291 (1987) 172.

[43] H. Ooguri, J. Rahmfeld, H. Robins, J. Tannenhauser, Holography in Superspace, JHEP 0007 (2000) 045, hep-th/0007104.

[44] L. Bonora, K. Lechner, M. Bregola, P. Pasti and M. Tonin, A Discussion of the Constraints in $N=1$ SUGRA-SYM in 10D, Int. J. Mod. Phys. A 5 (1990) 461.

[45] P. S. Howe, Pure spinors lines in superspace and ten-dimensional supersymmetric theories, Phys. Lett. B 258 (1991) 141 [Addendum-ibid. B 259 (1991) 511].

[46] P. S. Howe, Pure spinors, function superspaces and supergravity theories in ten-dimensions and eleven-dimensions, Phys. Lett. B 273 (1991) 90.

[47] E. Witten, On flux quantization in M-theory and the effective action, J. Geom. Phys. 22 (1997) 1 [arXiv:hep-th/9609122].

[48] N. Berkovits, C. Vafa and E. Witten, Conformal field theory of AdS background with Ramond-Ramond flux, JHEP 9903 (1999) 018 [arXiv:hep-th/9902098].

[49] N. Berkovits, Relating the RNS and pure spinor formalisms for the superstring, JHEP 0108, 026 (2001) [arXiv:hep-th/0104247]. 
[50] P. A. Grassi, G. Policastro and P. van Nieuwenhuizen, On the BRST cohomology of superstrings with / without pure spinors, Adv. Theor. Math. Phys. 7, 499 (2003) [arXiv:hep-th/0206216].

[51] P.A. Grassi, G. Policastro, P. van Nieuwehnuizen, in preparation.

[52] F. Sugino and P. Vanhove, U-duality from matrix membrane partition function, Phys. Lett. B 522, 145 (2001) [arXiv:hep-th/0107145].

[53] S. Naito, K. Osada and T. Fukui, Fierz Identities and Invariance of 11-dimensional Supergravity Action, Phys. Rev. D34 (1986) 536. 